\title{
Secrecy Outage Analysis for Cooperative NOMA Systems with Relay Selection Schemes
}

\author{
Hongjiang Lei, Member, IEEE, Zixuan Yang, Ki-Hong Park, Member, IEEE, Imran Shafique \\ Ansari, Member, IEEE, Yongcai Guo, Gaofeng Pan, Member, IEEE, \\ and Mohamed-Slim Alouini, Fellow, IEEE
}

\begin{abstract}
This paper considers the secrecy outage performance of a multiple-relay assisted non-orthogonal multiple access (NOMA) network over Nakagami- $m$ fading channels. Two time slots are utilized to transmit signals from the base station to destination. At the first time slot, the base station broadcasts the superposition signal of the two users to all decode-and-forward relays by message mapping strategy. Then the selected relay transmits superposition signal to the two users via power-domain NOMA technology. Three relay selection schemes, i.e., optimal single relay selection (OSRS) scheme, two-step single relay selection (TSRS) scheme, and optimal dual relay selection (ODRS) scheme, are proposed and the secrecy outage performance are analyzed. As a benchmark, we also examine the secrecy outage performance of the NOMA systems with traditional multiple relay forwarding (TMRF) scheme in which all the relay that successfully decode signals from the source forward signals to the NOMA users with equal power. Considering the correlation between the secrecy capacity of two users and different secrecy requirement for two NOMA users, the analytical expressions for the security outage probability (SOP) of the proposed OSRS, TSRS, and ODRS schemes along with the TMRF scheme are derived and validated via simulations. To get more insights, we also derive the analytical expressions for the asymptotic SOP for all the schemes with fixed and dynamic power allocations. Furthermore, the secrecy diversity order (SDO) and secrecy array gain of cooperative NOMA systems are obtained. The results demonstrate that our proposed schemes can significantly enhance the secrecy performance compared to the TMRF scheme and that all the schemes with fixed power allocation obtain zero SDO and the OSRS scheme with dynamic power allocation obtains the same SDO as TMRF.
\end{abstract}

Index Terms-Non-orthogonal multiple access, cooperative

Manuscript received.

This work was supported by the Project of Fundamental Science and Frontier Technology Research Plan of Chongqing under Grant cstc2017jcyjAX0204 and the Scientific and Technological Research Program of Chongqing Municipal Education Commission under Grant KJ1704088.

$\mathrm{H}$. Lei is with the School of Communication and Information Engineering, Chongqing University of Posts and Telecommunications, Chongqing 400065, China, and also with Shaanxi Key Laboratory of Information Communication Network and Security, Xi'an University of Posts \& Telecommunications, Xi'an, Shaanxi 710121, China (e-mail: leihj@cqupt.edu.cn).

Z. Yang is with the School of Communication and Information Engineering, Chongqing University of Posts and Telecommunications, Chongqing 400065, China.

I. S. Ansari is with the School of Engineering, University of Glasgow, Glasgow G12 8QQ, United Kingdom.

Y. Guo is with the Key Laboratory of Optoelectronic Technology and Systems of Ministry of Education of China, Chongqing University, Chongqing 400044, China.

G. Pan is with the School of Information and Electronics Engineering, Beijing Institute of Technology, Beijing 100081, China.

K.-H. Park and M.-S. Alouini are with CEMSE Division, King Abdullah University of Science and Technology (KAUST), Thuwal 23955-6900, Saudi Arabia. communication systems, relay selection scheme, secrecy outage probability

\section{INTRODUCTION}

\section{A. Background and Related Works}

Non-orthogonal multiple access (NOMA) has been considered as one of the most promising technologies to deal with the shortage of bandwidth resources and enhance user fairness in the fifth generation (5G) mobile network [1]-[6]. Compared to traditional orthogonal multiple access schemes, NOMA systems can obtain superior merits, such as high bandwidth efficiency, better user fairness, ultra-connectivity, and high flexibility. After receiving the superposition signal transmitted by the source node, the stronger user in NOMA systems firstly decodes the signal sent to the weaker user, cancels it from the received signals, and then decodes the signals for itself.

Cooperative communication is a particularly attractive technique that not only extends the network's coverage but also enhances the system performance when diversity technology is utilized at the destinations. Since the stronger users in NOMA systems always firstly decode the messages for the weaker users, therefore the stronger users can be utilized as relays to improve the performance of those weaker users with poor channel conditions [7], [8]. Ding et al. analyzed the outage probability (OP), diversity order of cooperative NOMA systems, and proposed an approach based on user pairing to reduce system complexity in [7]. Furthermore, a new cooperative simultaneous wireless information and power transfer NOMA protocol was proposed and the closed-form expressions for the OP and system throughput were derived in [8]. Recently, many literatures focused on the cooperative NOMA systems with dedicated relay nodes. For example, a dedicated amplify-and-forward (AF) relay with multiple antennas was utilized in the cooperative NOMA systems and the closed-form expressions for the exact and lower bound of OP were derived in [9], [10], [11]. Moreover, a novel power allocation (PA) scheme for dual-hop relaying NOMA systems along with its ergodic sum-rate and OP were investigated in [12]. In [13] and [14], Lv et al. discussed cognitive cooperative NOMA systems, in which a cognitive user (stronger user) was utilized as a relay to primary users (weaker users).

Full-duplex (FD) relay also has been utilized in cooperative NOMA systems to obtain higher spectral efficiency and better performance [15]. For example, the stronger user working in the FD mode was utilized to forward signals to the weaker 
user in [16] and [17]. The closed-form expression for OP was obtained and the results showed that FD relays outperform half-duplex (HD) relays. Liu et al. analyzed the PA problems for HD and FD cooperative NOMA systems thereby obtaining the closed-form expression for the optimal power allocation policies in [17]. Since the weaker user decodes its signals by directly treating signals for the stronger user as interference, then the performance of the weaker users will become the bottleneck of NOMA systems in many scenarios. The dedicated FD relay was utilized to improve the performance of the weaker users in [18], [19]. The performance of FD cooperative NOMA systems over Nakagami- $m$ channels was investigated in [18] and the analytical expressions for OP and ergodic rate were derived. In [19], a sharing FD relay was utilized in NOMA systems with two source-destination pairs and the ergodic rate, OP, and outage capacity were investigated while both perfect and imperfect self-interference cancellation (SIC) schemes were considered, respectively.

Relay selection (RS) technique is an effective scheme in making full use of space diversity with low implementation complexity and it can straightforwardly improve the spectral efficiency of cooperative systems [20] - [25]. Considering the quality of service $(\mathrm{QoS})$ requirements for the two users are different, a two-stage single-relay-selection strategy and dualrelay-selection with fixed power allocation (FPA) was studied in [20] and [21], respectively, in order to obtain the OP. The results showed that the two-stage strategy achieves a better OP and array gain. Combining DF and AF relaying, Yang et al. extended the two-stage RS scheme with dynamic power allocation (DPA) and derived the exact and asymptotic analytical expressions for OP in [22]. In [23], considering whether the relay can correctly decode two users' messages, two optimal RS schemes were proposed for cooperative NOMA systems with FPA and DPA at the relays, respectively. Yue et al., in [24], studied the performance of cooperative NOMA systems with two RS schemes in which the relay operates in either FD or HD mode. Modeling the spatial topology of relays with homogeneous poisson point process (PPP), the performance of cooperative NOMA systems with two-stage RS scheme was analyzed in [25] and a closed-form approximation for the OP was obtained.

To deal with the security issues followed by the explosive increase in cellular data, physical layer security (PLS) is emerging as one of the most promising ways to ensure secure communication basing on the time varying nature of the wireless channels [26].A new optimal PA strategy was proposed to maximize the secrecy sum rate of NOMA systems and the results confirmed that a significant secrecy performance for NOMA systems was obtained in [27]. In [28], the security performance of a NOMA-based large-scale network is considered, where both the NOMA users and eavesdroppers were modeled by homogeneous PPPs. In [29], we investigated the secrecy outage performance of a multiple-input single-output (MISO) NOMA systems with transmit antenna selection (TAS) schemes and the closed-form expressions for secrecy outage probability (SOP) were derived. The results demonstrated that the proposed DPA scheme can achieve non-zero secrecy diversity order (SDO) for all the TAS schemes. The secrecy outage performance of multiple-input multiple-output (MIMO) NOMA systems with multiple legitimate and illegitimate receivers was studied in [30] and the closed-form expressions for SOP were subsequently obtained. Feng et al. proposed in [31] a new PA to maximize the secrecy rate of the stronger user while guaranteeing the non-secure transmission rate requirement to the weaker user, but the secrecy performance of the weaker user was not considered. Secrecy beamforming schemes were proposed for MISO-NOMA systems, cognitive MISO-NOMA systems, and MIMO-NOMA systems in [32], [33], [34], and [35], respectively. The secrecy performance of a NOMA system with multiple eavesdroppers was investigated while zero-forcing and minimum mean-square error decoding schemes were utilized on the legitimate destinations in [36]. A new joint subcarrier (SC) assignment and PA scheme was proposed to improve the security of the two-way relay NOMA systems in [37].

The secrecy performance of a cooperative NOMA system with a dedicated AF/DF relay was investigated and the closedform expressions for the SOP were obtained in [38]. In some scenarios, the relay in cooperative NOMA systems is untrusted and curious to decode the users' messages. Two new HD nonorthogonal AF schemes were proposed for cooperative NOMA systems with an untrusted relay and the secrecy rate maximization-based PA scheme was discussed in [39]. Arafa et al. proposed two new relaying schemes to resist the untrusted relay and the secrecy performance of cooperative NOMA systems with new relaying schemes was analyzed and compared in [40]. Feng et al. proposed an artificial-noise (AN) aided scheme to build up secure cooperative NOMA systems with a FD relay and the closed-form expression for the SOP was derived in [41]. The secrecy performance of cooperative NOMA systems in which the stronger user behaves as a FD relay was investigated in [42] and the SOP was analyzed with an assumption of the imperfect SIC. The security-reliability tradeoff for both cooperative and non-cooperative NOMA schemes was analyzed and analytical expressions for SOP were derived in [43].

Furthermore, RS schemes were utilized to enhance the secrecy performance of cooperative systems [44] - [48]. In [44], Zou et al. proposed new optimal relay selection schemes for AF and DF cooperative wireless networks. The closedform expressions for the intercept probability were derived and compared with traditional multiple relay forwarding scheme. Several different selection criteria were presented to enrich the secrecy performance of multi-AF cooperative communication systems in [45] and [46], respectively, in which the co-channel interference and correlated fading channels were considered. Moreover, the secrecy performance of cognitive DF relay systems was investigated in [47] and [48], respectively, in which the independent and identically distributed (i.i.d.) and independent but not necessarily identical distributed (i.n.i.d.) quasi-static Nakagami- $m$ fading was considered.

\section{B. Motivation and Contributions}

In many works, such as [28], [30], [38], the worst-case scenario was considered, in which it is assumed that the 
eavesdroppers have powerful detection capabilities and they can extract the message from the signals transmitted from the resource. Under this assumption, one can realize that the secrecy capacity of all the users in NOMA systems are not independent [28], [30]. As mentioned above, the secrecy performance of NOMA systems has been studied in various scenarios, but there is no open literature considering the dependence between the secrecy capacity of NOMA users. Furthermore, the researchers proposed many different RS schemes to improve the general performance (such as OP) of the NOMA systems, but all these RS schemes cannot be directly utilized to enhance the secrecy performance of NOMA systems. It must be noted that the interdependency between NOMA users makes the performance analysis of cooperative NOMA systems more complicated and challenging.

The major contributions of this paper are as follows ${ }^{1}$

1) In this work, we analyze a cooperative NOMA system with multiple relays and an eavesdropper employing RS schemes to enhance the secrecy performance. Three RS (OSRS, TSRS, and ODRS) schemes are proposed. For the purpose of comparison, we also investigate the secrecy performance of the NOMA systems with traditional multiple relay forwarding (TMRF) scheme in which all the relays that successfully decode signals from the source forward signals to the NOMA users with equal power. Considering the correlation between the secrecy capacity of two users, the analytical expressions for the SOP under different RS schemes are derived and validated via simulations.

2) Different secrecy QoS is considered in our work, i.e., different secrecy threshold rates are requested for the two NOMA users. In NOMA systems, the stronger user performs SIC to delete the signals sent for the weaker user, while the weaker user views the signals sent for the stronger user as interference. Thus, the signal-to-interference-noise ratio (SINR) of the weaker user is usually less than the signal to noise ratio (SNR) of stronger user. When the secrecy QoS requirements for both users are same, then the weaker user is the bottleneck of the NOMA systems. However, in the scenarios that the secrecy QoS requirements for both users are different, either the stronger or the weaker user will be the bottleneck of the cooperative NOMA systems.

3) To obtain more insights, we also derive the analytical expressions for the asymptotic SOP under different RS schemes with FPA and DPA. Furthermore, the SDO and secrecy array gain (SAG) of cooperative NOMA systems are derived. The results demonstrate that all the RS schemes with FPA obtain zero SDO and while the RS schemes with DPA obtain non-zero SDO. Moreover, the OSRS scheme with DPA achieves the same SDO as TMRF scheme with DPA.

\footnotetext{
${ }^{1}$ A preliminary result of this work has been presented in [49], where we analyzed secrecy performance of multiple-relay assisted NOMA systems over Rayleigh channels. Whereas, in this paper, we investigate secrecy outage performance of cooperative NOMA systems with relay selection schemes over Nakagami- $m$ channels and more RS schemes are proposed.
}

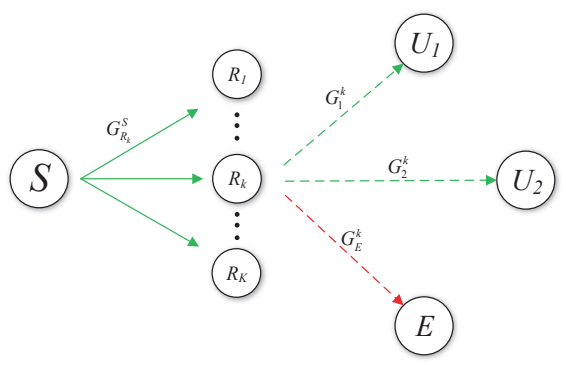

Fig. 1. System model demonstrating a cooperative NOMA system with one source $(S)$, multiple relays $\left(R_{k}, k=1, \cdots, K\right)$, two legitimate users $\left(U_{1}\right.$ and $U_{2}$ ), and an illegitimate eavesdropper $(E)$.

4) Differing from [28], [30], [38], wherein it has been assumed that the secrecy capacities for the stronger and weaker users are independent, we consider correlation between the secrecy capacity of the two users and different secrecy requirement for the two users in this work. Technically speaking, it is much more challenging and more practical to analyze the secrecy performance when the dependence is considered.

\section{Organization}

This paper is organized as follows, in Section II the system model and RS schemes are introduced. In Section III], we analyze the security outage performance of the proposed system. The asymptotic SOP for cooperative NOMA systems with FPA and DPA are derived in Sections IV and $\mathrm{V}$, respectively. Section VI summarizes the difference among all the proposed schemes. Numerical and simulation results are presented in Section VII to demonstrate the security performance of the system. Finally, Section VIII concludes the paper.

\section{SYSTEM MODEL}

As shown in Fig. 1] we consider a cooperative downlink NOMA system that consists of a base station $(S), K(K \geq 1)$ DF HD relays $\left(R_{k}, k=1, \cdots, K\right)$, and two users ( $U_{1}$ and $\left.U_{2}\right)$. An eavesdropper $(E)$ wants to wiretap the information through decoding the received signals. It is assumed that all nodes are equipped with a single antenna and the direct link between $S$ and both users/eavsesdropper are unavailable due to deep fading or other unknown reasons. The communication links between $S$ and all the receivers $\left(U_{1}, U_{2}\right.$, and $E$ ) are relayed by $R_{k}$.

All the channels are assumed to undergo independent and slow Nakagami- $m$ fading model and remain unchanged during each fading block but independently vary from one block to another. Then the probability density function (PDF) and the cumulative distribution function (CDF) of the channel power gains of link between the node $\hbar$ and the node $\lambda$ can be expressed by

$$
\begin{gathered}
f_{G_{\lambda}^{\hbar}}(x)=\beta_{\lambda}^{\hbar} x^{m_{\lambda}^{h}-1} e^{-\lambda_{\lambda}^{h} x}, \\
F_{G_{\lambda}^{\hbar}}(x)=1-e^{-\lambda_{\lambda}^{\hbar} x} \sum_{k=0}^{m_{\lambda}^{\hbar}-1} \frac{\left(\lambda_{\lambda}^{\hbar}\right)^{k}}{k !} x^{k},
\end{gathered}
$$


where $\hbar \in\left\{S, R_{k}\right\}$ signifies the transmitter, $\lambda \in$ $\left\{R_{k}, U_{1}, U_{2}, E\right\}$ denotes the receiver, $\beta_{\lambda}^{\hbar}=\frac{\left(\lambda_{\lambda}^{\hbar}\right)^{m_{\lambda}^{\hbar}}}{\Gamma\left(m_{\lambda}^{\hbar}\right)}, \lambda_{\lambda}^{\hbar}=$ $\frac{m_{\lambda}^{\hbar}}{\Omega_{\lambda}^{\hbar}}, \Gamma(\cdot)$ is Gamma function, as defined by (8.310.1) of [50], $m_{\lambda}^{\hbar}$ denotes the fading parameters and is assumed to be integer, and $\Omega_{\lambda}^{\hbar}$ means average channel power gains.

To make analysis simple, it is assumed that the links of the first hop are i.i.d., which means $m_{R_{k}}^{S}=m_{R}, \Omega_{R_{k}}^{S}=\Omega_{R}$. The same assumption is made to the links between $R_{k}$ and each receiver in the second hop, which means $m_{U_{1}}^{R_{k}}=m_{U_{2}}^{R_{k}}=m_{U}$, $\Omega_{U_{1}}^{R_{k}}=\Omega_{1}, \Omega_{U_{2}}^{R_{k}}=\Omega_{2}, m_{E}^{R_{k}}=m_{E}, \Omega_{E}^{R_{k}}=\Omega_{E}, \lambda_{U_{i}}^{R_{k}}=\lambda_{i}$, $i=1,2, \lambda_{R_{k}}^{S}=\lambda_{R}, \lambda_{E}^{R_{k}}=\lambda_{E}$, and $\beta_{E}^{R_{k}}=\beta_{E}$,

Similar to [11], [16], [18] - [24], it is assumed that there is perfect synchronization between all the relays and two time slots are utilized for the communication between $S$ and the NOMA users. In the first time slot, $S$ broadcasts the superposition signal to all relays by the message mapping strategy, which is proposed and proved optimal to achieve the minimal common OP in [23]. During the second time slot, a relay is selected using the RS scheme proposed below to send superposition signal to the two NOMA users. We assume all the relays are close enough such that the relative distance between the two users and the relay is determined.

To make the representation clear and simple, we use $G_{U_{i}}^{R_{k}}=$ $G_{i}^{k}$. It is also assumed that $U_{1}$ has a better channel condition than $U_{2}\left(\Omega_{1}>\Omega_{2}\right)$, which is adopted in many NOMA studies, e.g., [15], [16], [17], [23], [29]. Then the SINR/SNR of the NOMA users can be written as

$$
\begin{gathered}
\gamma_{1}^{k}=\alpha_{1} \rho_{R} G_{1}^{k}, \\
\gamma_{2}^{k}=\frac{\alpha_{2} \rho_{R} G_{2}^{k}}{\alpha_{1} \rho_{R} G_{2}^{k}+1},
\end{gathered}
$$

respectively, where $\alpha_{i}(i=1,2)$ represents the power allocation coefficients at the $k$ th relay, $\alpha_{1}+\alpha_{2}=1, \alpha_{1}<\alpha_{2}$, $\rho_{R}=\frac{P_{R}}{\sigma^{2}}$, where $P_{R}$ denotes the transmit power at $k$ th relay, and $\sigma^{2}$ signifies the noise power.

\section{Secrecy Outage Probability Analysis}

The set of relays that can correctly decode mixed signals from $S$ can be expressed as

$\Phi \triangleq\left\{R_{k} \in \Re: 1 \leq k \leq K, \frac{1}{2} \ln \left(1+\rho_{S} G_{R_{k}}^{S}\right) \geq R_{1}^{\mathrm{th}}+R_{2}^{\mathrm{th}}\right\}$,

where $\Re$ denotes the set of all the relays, the factor $\frac{1}{2}$ arises from the fact that two time slots are required to complete the data transmission, $\rho_{S}=\frac{P_{S}}{\sigma^{2}}, P_{S}$ denotes the transmit power at $S$, and $R_{i}^{\text {th }}(i=1,2)$ is the data rate threshold for $U_{i}$. Thus the SOP of cooperative NOMA systems can be written as

$$
P_{\text {out }}=\sum_{n=0}^{K} \operatorname{Pr}(|\Phi|=n) P_{\Phi_{n}},
$$

where $P_{\Phi_{n}}$ denotes the SOP under the condition that there are $n$ relays that correctly decode the mixed signals.
It is assumed that all the links between the source and the relays are i.i.d., so we have

$$
\begin{aligned}
\operatorname{Pr}\{|\Phi|=n\} & =C_{K}^{n}\left(\operatorname{Pr}\left\{G_{R_{k}}^{S} \geq \eta\right\}\right)^{n}\left(\operatorname{Pr}\left\{G_{R_{k}}^{S}<\eta\right\}\right)^{K-n} \\
& =C_{K}^{n} \chi^{n}(1-\chi)^{K-n},
\end{aligned}
$$

where $C_{m}^{n}=\frac{m !}{n !(m-n) !}, \eta=\frac{e^{2 R_{1}^{\mathrm{th}}+2 R_{2}^{\mathrm{th}}}-1}{\rho_{S}}$, and $\chi=$ $e^{-\lambda_{R} \eta} \sum_{k=0}^{m_{R}-1} \frac{\lambda_{R}^{k} \eta^{k}}{k !}$

\section{A. Traditional Multiple Relay Forwarding Scheme}

As a benchmark, the traditional multiple relay forwarding (TMRF) scheme is presented in this subsection, where all the relays can successfully decode and forward the signal to both the users with equal power. Both the legitimate and illegitimate receivers combine their received signals with maximal ratio combining (MRC) scheme to maximize their SINR ${ }^{2}$. To make fair comparison, it is assumed that the total transmit power at these relays is given by $P_{R}$.

One can easily have $\operatorname{Pr}\{|\Phi|=0\}=(1-\chi)^{K}$ and $P_{\Phi_{0}}=$ 1. In this subsection, it assumed that $n \geq 1$. Then the $P_{\Phi_{n}}$ with this scheme can be expressed as

$$
P_{\Phi_{n}}=\operatorname{Pr}\left\{C_{s, 1}^{\mathrm{TMRF}}<R_{1}^{s} \text { or } C_{s, 2}^{\mathrm{TMRF}}<R_{2}^{s}|| \Phi \mid=n\right\},
$$

where $C_{s, i}^{\mathrm{TMRF}}=\frac{1}{2} \ln \left(\frac{1+\gamma_{i}^{\mathrm{TMRF}}}{1+\gamma_{E}^{\mathrm{TMRF}}}\right)$ means the secrecy capacity of $U_{i}, i=1,2, \gamma_{1}^{\mathrm{TMRF}}=\rho_{1} \alpha_{1} G_{1}^{\mathrm{TMRF}}, \gamma_{2}^{\mathrm{TMRF}}=$ $\frac{\rho_{1} \alpha_{2} G_{2}^{\mathrm{TMRF}}}{1+\rho_{1} \alpha_{1} G_{2}^{\mathrm{TMRF}}}, G_{i}^{\mathrm{TMRF}}=\sum_{k=1}^{n} G_{i}^{k}, \gamma_{E, i}^{\mathrm{TMRF}}$ signifies the SNR at $E$ when $U_{i}$ is wiretapped, $R_{i}^{s}$ is the secrecy rate threshold for $U_{i}, \rho_{1}=\frac{P_{R}}{n \sigma^{2}}$, and $\sigma^{2}$ is the noise power.

The CDF of $G_{i}^{\mathrm{TMRF}}$ can be expressed as [51]

$$
F_{G_{i}^{\mathrm{TMRF}}}(x)=1-e^{-\lambda_{i} x} \sum_{k=0}^{\tau_{U}-1} \frac{\lambda_{i}^{k}}{k !} x^{k},
$$

where $i=1,2$ and $\tau_{U}=n m_{U}$.

As similar to [28], [30], and [38], we assume that $E$ has enough capabilities to detect multiuser data. Then the SNR at $E$ can be expressed as

$$
\gamma_{E, i}^{\mathrm{TMRF}}=\rho_{1} \alpha_{i} G_{E}^{\mathrm{TMRF}},
$$

where $i=1,2$ and $G_{E}^{\mathrm{TMRF}}=\sum_{k=1}^{n} G_{E}^{k}$. Similarly, the PDF of $G_{E}^{\mathrm{TMRF}}$ can be expressed as [51]

$$
f_{G_{E}^{\mathrm{TMRF}}}(x)=\beta_{E}^{\mathrm{TMRF}} x^{\tau_{E}-1} e^{-\lambda_{E} x},
$$

where $\beta_{E}^{\mathrm{TMRF}}=\frac{\lambda_{E}^{\tau_{E}}}{\Gamma\left(\tau_{E}\right)}$ and $\tau_{E}=n m_{E}$.

We can express the secrecy connection probability (SCP) of $U_{1}$, which is the complementary of SOP, as

$$
\operatorname{Pr}\left\{C_{s, 1}^{\mathrm{TMRF}}>R_{1}^{s}\right\}=\operatorname{Pr}\left\{G_{1}^{\mathrm{TMRF}}>\delta_{1}\left(G_{E}^{\mathrm{TMRF}}\right)\right\},
$$

where $\delta_{1}(x)=b_{1}+\theta_{1} x, b_{1}=\frac{\theta_{1}-1}{\alpha_{1} \rho_{1}}$, and $\theta_{1}=e^{2 R_{1}^{s}}$.

${ }^{2}$ It is ideally assumed that there is a space time code (such as orthogonal space-time block coding) realized at all the relays then the MRC scheme can be utilized at all the users to maximize their SINRs. Specific design of space time coding is out of scope of this work. 


$$
\begin{aligned}
\operatorname{Pr}\left\{C_{s, 2}^{\mathrm{TMRF}}>R_{1}^{s}\right\} & =\operatorname{Pr}\left\{\rho_{1} G_{2}^{\mathrm{TMRF}}(\underbrace{1-\theta_{2} \alpha_{1}-\theta_{2} \alpha_{1} \alpha_{2} \rho_{1} G_{E}^{\mathrm{TMRF}}}_{\triangleq_{\Lambda}})>\theta_{2}-1+\theta_{2} \alpha_{2} \rho_{1} G_{E}^{\mathrm{TMRF}}\right\} \\
& =\operatorname{Pr}\left\{\Lambda>0, G_{2}^{\mathrm{TMRF}}>\delta_{2}\left(G_{E}^{\mathrm{TMRF}}\right)\right\} \\
& =\operatorname{Pr}\left\{G_{E}^{\mathrm{TMRF}}<a_{1}, G_{2}^{\mathrm{TMRF}}>\delta_{2}\left(G_{E}^{\mathrm{TMRF}}\right)\right\}
\end{aligned}
$$

$$
\begin{aligned}
P_{\Phi_{n}}^{\mathrm{TMRF}} & =\operatorname{Pr}\left\{\left(C_{s, 1}^{\mathrm{TMRF}}<R_{1}^{s} \text { or } C_{s, 2}^{\mathrm{TMRF}}<R_{2}^{s}\right) \| \Phi \mid=n\right\} \\
& =1-\operatorname{Pr}\left\{G_{1}^{\mathrm{TMRF}}>\delta_{1}\left(G_{E}^{\mathrm{TMRF}}\right), G_{2}^{\mathrm{TMRF}}>\delta_{2}\left(G_{E}^{\mathrm{TMRF}}\right), G_{E}^{\mathrm{TMRF}}<a_{1}\right\} \\
& =1-\int_{0}^{a_{1}} \operatorname{Pr}\left\{G_{1}^{\mathrm{TMRF}}>\delta_{1}(x)\right\} \operatorname{Pr}\left\{G_{2}^{\mathrm{TMRF}}>\delta_{2}(x)\right\} f_{G_{E}^{\mathrm{TMRF}}}(x) d x \\
& =1-\int_{0}^{a_{1}}\left(1-F_{G_{1}^{\mathrm{TMRF}}}\left(\delta_{1}(x)\right)\right)\left(1-F_{G_{2}^{\mathrm{TMRF}}}\left(\delta_{2}(x)\right)\right) f_{G_{E}^{\mathrm{TMRF}}}(x) d x
\end{aligned}
$$

$$
\begin{aligned}
P_{\text {out }, 2} & =1-\operatorname{Pr}\left\{G_{E}^{m}<a_{2}, G_{2}^{m}>\delta_{4}\left(G_{E}^{m}\right)\right\} \\
& =1-\left(\operatorname{Pr}\left\{G_{E}^{m}<a_{2}\right\}-\operatorname{Pr}\left\{G_{E}^{m}<a_{2}, G_{2}^{m}<\delta_{4}\left(G_{E}^{m}\right)\right\}\right) \\
& =\operatorname{Pr}\left\{G_{E}^{m}<a_{2}, G_{2}^{m}<\delta_{4}\left(G_{E}^{m}\right)\right\}+1-\operatorname{Pr}\left\{G_{E}^{m}<a_{2}\right\} \\
& =\operatorname{Pr}\left\{G_{2}^{m}<\delta_{4}\left(G_{E}^{m}\right) \mid G_{E}^{m}<a_{2}\right\} \operatorname{Pr}\left\{G_{E}^{m}<a_{2}\right\}+\operatorname{Pr}\left\{G_{E}^{m}>a_{2}\right\} \\
& =\operatorname{Pr}\left\{\frac{G_{2}^{m}}{\delta_{4}\left(G_{E}^{m}\right)}<1 \mid G_{E}^{m}<a_{2}\right\} \operatorname{Pr}\left\{G_{E}^{m}<a_{2}\right\}+\operatorname{Pr}\left\{G_{E}^{m}>a_{2}\right\}
\end{aligned}
$$

Similarly, we obtain the SCP of $U_{2}$ as $(13)$, given at the top of this page, where $a_{1}=\frac{1-\theta_{2} \alpha_{1}}{\rho_{1} \alpha_{1} \alpha_{2} \theta_{2}}, \delta_{2}(x)=c_{1}+\frac{\alpha_{2}}{d_{1}-e_{1} x}$, $c_{1}=-\frac{1}{\alpha_{1} \rho_{1}}, d_{1}=\alpha_{1} \rho_{1}\left(1-\alpha_{1} \theta_{2}\right), e_{1}=\rho_{1}^{2} \alpha_{1}^{2} \alpha_{2} \theta_{2}$, and $\theta_{2}=e^{2 R_{2}^{s}}$.

Remark 1: One can easily find that secrecy outage would occur at $U_{2}$ when $1-\theta_{2} \alpha_{1}<0$. This means that in order to ensure a secure NOMA system $\left(U_{2}\right)$, there is a constraint for the power allocation coefficients, which is expressed as

$$
\alpha_{1} \leq \frac{1}{\theta_{2}}=e^{-2 R_{2}^{s}} \text { or } \alpha_{2}>1-\frac{1}{\theta_{2}}=1-e^{-2 R_{2}^{s}} .
$$

Based on (12) and (13), $P_{\Phi_{n}}$ in this case can be rewritten as (15), given at the top of this page.

To facilitate the following analysis, we define

$$
\begin{aligned}
g(a, b, c, r, q, f, h, k, j) & =\int_{0}^{a} x^{b-1} e^{-f x-\frac{h}{1-q x}} \\
& \times(1+c x)^{k}\left(1+\frac{r}{1-q x}\right)^{j} d x .
\end{aligned}
$$

To the authors' best knowledge, it's very difficult to obtain the closed-form expression of $g(a, b, c, r, q, f, h, k, j)$. Here by making use of Gaussian-Chebyshev quadrature from eq. (25.4.30) of [52], we obtain the approximation for $g(a, b, c, r, q, f, h, k, j)$ as

$$
\begin{aligned}
g(a, b, c, r, q, f, h, k, j) & \approx\left(\frac{a}{2}\right)^{b} \sum_{i=1}^{N} w_{i} s_{i}^{b-1} e^{-\frac{a f s_{i}}{2}-\frac{2 h}{2-a q s_{i}}} \\
& \times\left(1+\frac{a c s_{i}}{2}\right)^{k}\left(1+\frac{2 r}{2-a q s_{i}}\right)^{j},
\end{aligned}
$$

where $N$ is the number of terms, $s_{i}=t_{i}+1, t_{i}$ is the $i$ th zero of Legendre polynomials, $w_{i}$ is the Gaussian weight, which is given in Table (25.4) of [52].

Substituting (9) and (11) into (15) and with some simple algebraic manipulations, we have

$$
P_{\Phi_{n}}^{\mathrm{TMRF}}=1-\beta_{E}^{\mathrm{TMRF}} e^{-\lambda_{1} b_{1}-\lambda_{2} c_{1}} \sum_{k=0}^{\tau_{U}-1} \sum_{j=0}^{\tau_{U}-1} \frac{\lambda_{1}^{k} \lambda_{2}^{j} \Xi_{1}}{k ! j !},
$$

where $\Xi_{1}=b_{1}^{k} c_{1}^{j} g\left(a_{1}, \tau_{E}, \frac{\theta_{1}}{b_{1}}, \frac{\alpha_{2}}{d_{1} c_{1}}, \frac{e_{1}}{d_{1}}, \lambda_{1} \theta_{1}+\lambda_{E}, \frac{\lambda_{2} \alpha_{2}}{d_{1}}, k, j\right)$.

\section{B. Optimal Single Relay Selection Scheme}

In this subsection, we propose OSRS scheme to minimize the overall SOP of the proposed cooperative NOMA system 3 Based on (12), one can easily obtain the SOP for $U_{1}$ with $m$ th relay as

$$
\begin{aligned}
P_{\text {out }, 1} & =1-\operatorname{Pr}\left\{G_{1}^{m}>\delta_{3}\left(G_{E}^{m}\right)\right\} \\
& =\operatorname{Pr}\left\{G_{1}^{m}<\delta_{3}\left(G_{E}^{m}\right)\right\} \\
& =\operatorname{Pr}\left\{\frac{G_{1}^{m}}{\delta_{3}\left(G_{E}^{m}\right)}<1\right\},
\end{aligned}
$$

where $\delta_{3}(x)=b_{2}+\theta_{1} x, b_{2}=\frac{\theta_{1}-1}{\alpha_{1} \rho_{2}}$, and $\rho_{2}=\frac{P_{R}}{\sigma^{2}}$.

Similarly, based on (13), we can express SOP for $U_{2}$ as 20 , given at the top of this page, where $a_{2}=\frac{1-\theta_{2} \alpha_{1}}{\theta_{2} \alpha_{1} \alpha_{2} \rho_{2}}$, $\delta_{4}(x)=c_{2}+\frac{\alpha_{2}}{d_{2}-e_{2} x}, c_{2}=-\frac{1}{\alpha_{1} \rho_{2}}, d_{2}=\alpha_{1} \rho_{2}-\theta_{2} \alpha_{1}^{2} \rho_{2}$,

\footnotetext{
${ }^{3}$ In this work, it is assumed that the source node has CSI of the eavesdropping links, which is a common assumption in the physical layer security literature. The CSI of the eavesdropping links can be estimated and obtained by monitoring the eavesdropper's transmissions or with some sophisticated channel estimation algorithms, which have been introduced in [26], [53], and [54].
} 


$$
\begin{aligned}
P_{\Phi_{n}}^{\text {OSRS }} & =\left(\operatorname{Pr}\left\{X_{m}<1\right\}\right)^{n} \\
& =\left(1-\operatorname{Pr}\left\{X_{m}>1\right\}\right)^{n} \\
& =\left(1-\operatorname{Pr}\left\{\min \left\{\frac{G_{1}^{m}}{\delta_{3}\left(G_{E}^{m}\right)}, \frac{G_{2}^{m}}{\delta_{4}\left(G_{E}^{m}\right)}\right\} \geq 1, G_{E}^{m}<a_{2}\right\}\right)^{n} \\
& =(1-\underbrace{\operatorname{Pr}\left\{G_{1}^{m} \geq \delta_{3}\left(G_{E}^{m}\right), G_{2}^{m} \geq \delta_{4}\left(G_{E}^{m}\right), G_{E}^{m}<a_{2}\right\}}_{\triangleq_{\Delta_{1}}})^{n}
\end{aligned}
$$

and $e_{2}=\theta_{2} \alpha_{1}^{2} \alpha_{2} \rho_{2}^{2}$. One can observe that when $G_{E}^{m}>a_{2}$ the secrecy outage would occur at $U_{2}$, which means the cooperative NOMA system is not secure.

For the $m$ th relay, we define

$$
X_{m}=\left\{\begin{array}{cc}
\min \left\{\frac{G_{1}^{m}}{\delta_{3}\left(G_{E}^{m}\right)}, \frac{G_{2}^{m}}{\delta_{4}\left(G_{E}^{m}\right)}\right\}, & G_{E}^{m}<a_{2} \\
0, & G_{E}^{m} \geq a_{2}
\end{array} .\right.
$$

Then to maximize the secrecy performance the relay is selected with the following criterion

$$
m^{*}=\arg \max _{m \in \Phi}\left(X_{m}\right) .
$$

Remark 2: It should be noted that the selection scheme in (22) is different from the max-min transmit antenna selection scheme (MMTAS) proposed in [30], which is to maximize the minimum secrecy capacity of two users. Here the selection criterion is to minimize the SOP of the cooperative NOMA system.

Remark 3: There is another important difference between MMTAS and OSRS, i.e., it is assumed that $R_{1}^{s}=R_{2}^{s}$ in MMTAS. However, in the OSRS scheme, $R_{1}^{s}$ and $R_{2}^{s}$ can be different. MMTAS is a special case of OSRS when it is assumed that $R_{1}^{s}=R_{2}^{s}$ and the secrecy capacities of the two users are independent.

The SOP of the cooperative NOMA system conditioned on $|\Phi|=n$ can be written as 23), given at the top of this page.

Based on (12) and (13), we have

$$
\begin{aligned}
\Delta_{1} & =\operatorname{Pr}\left\{G_{1}^{m} \geq \delta_{3}\left(G_{E}^{m}\right), G_{2}^{m} \geq \delta_{4}\left(G_{E}^{m}\right), G_{E}^{m}<a_{2}\right\} \\
& =\int_{0}^{a_{2}}\left(1-F_{G_{1}^{m}}\left(\delta_{3}(x)\right)\right)\left(1-F_{G_{2}^{m}}\left(\delta_{4}(x)\right)\right) f_{G_{E}^{m}}(x) d x \\
& =\beta_{E} e^{-\lambda_{1} b_{2}-\lambda_{2} c_{2}} \sum_{k=0}^{m_{U}-1} \frac{\lambda_{1}^{k}}{k !} \sum_{j=0}^{m_{U}-1} \frac{\lambda_{2}^{j}}{j !} \Xi_{2},
\end{aligned}
$$

where $\Xi_{2}=b_{2}^{k} c_{2}^{j} g\left(a_{2}, m_{E}, \frac{\theta_{1}}{b_{2}}, \frac{\alpha_{2}}{d_{2} c_{2}}, \frac{e_{2}}{d_{2}}, \lambda_{1} \theta_{1}+\lambda_{E}, \frac{\lambda_{2} \alpha_{2}}{d_{2}}, k, j\right)$.

\section{Two-Step Single Relay Selection Scheme}

In some scenarios stated in [20], [21], the QoS requirements for the two users are different. We can easily obtain the similar conclusion: the secrecy QoS for one user is higher than that of the other user. In this subsection, a new two-step RS (TSRS) scheme is proposed for such scenarios and the follwing two purposes will be realized simultaneously. One is to ensure there is no secrecy outage for the user that has lower secrecy QoS requirement and the other is to serve the user with higher secrecy QoS requirement with a secrecy rate as large as possible. Here we assume that the secrecy QoS requirement of $U_{2}$ is higher than that of $U_{1}$. The TSRS scheme is presented as follows.

In the first step, the following subset is built in the relays by focusing on $U_{1}$ 's target secrecy rate

$$
\Psi=\left\{i: i \in \Phi, C_{s, 1}^{i}>R_{1}^{s}\right\},
$$

where $C_{s, 1}^{i}=\frac{1}{2} \ln \left(\frac{1+\alpha_{1} \rho_{2} G_{1}^{i}}{1+\alpha_{1} \rho_{2} G_{E}^{i}}\right)$ signifying the secrecy capacity for $U_{1}$ from $R_{i}$ and $i \in \Phi$.

At the second step, the relay to maximize the secrecy capacity of $U_{2}$ is selected, i.e.,

$$
j^{*}=\arg \max _{j \in \Psi}\left(C_{s, 2}^{j}\right),
$$

where $C_{s, 2}^{j}=\frac{1}{2} \ln \left(\frac{1+\frac{\alpha_{2} \rho_{2} G_{2}^{j}}{\alpha_{1} \rho_{2} G_{2}^{j}+1}}{1+\alpha_{2} \rho_{2} G_{E}^{j}}\right)$ signifies the secrecy capacity for $U_{2}$ from $R_{j}(j \in \Psi)$.

The SOP with this scheme can be achieved as (27), given at the top of the next page.

Remark 4: An interesting result can be observed from 27. that the expression for the SOP under TSRS scheme is the same as that under OSRS, which means that the priority based on secrecy QoS does not influence the secrecy performance of the cooperative NOMA systems. It is easy to understand since the NOMA systems are secure only when no secrecy outage occurs for both the users.

\section{Optimal Dual Relay Selection Scheme}

In this subsection, a new RS scheme named ODRS scheme is proposed, in which one relay is selected to transmit signals to NOMA users among the ones that can decode the mixed signals and another relay is selected to transmit AN among the ones that can not decode the source signals. In order to deteriorate the SINR at $E$, we select the jamming relay with the following criterion as

$$
k^{*}=\arg \max _{k \in \bar{\Phi}}\left(G_{E}^{k}\right),
$$

where $\bar{\Phi}$ means the complement of $\Phi$. For the sake of a fair comparison with TMRF and OSRS schemes, the total transmit power of all the relays is constrained to $P_{R}$. It is assumed that $\alpha_{\mathrm{J}}\left(0 \leq \alpha_{\mathrm{J}}<1\right)$ portion is utilized to transmit the jamming signals, then the SINR at $E$ can be written as

$$
\gamma_{E, i}^{\mathrm{J}}=\frac{\alpha_{i} \rho_{3} G_{E}^{k}}{1+\rho_{4} H_{E}},
$$




$$
\begin{aligned}
P_{\Phi_{n}}^{\mathrm{TSRS}} & =\sum_{j=0}^{n} C_{n}^{j} \operatorname{Pr}\left\{\min _{i \in \Psi,|\Psi|=j}\left\{C_{s, 1}^{i}\right\}>R_{1}^{s}, \max _{k \in \bar{\Psi},|\bar{\Psi}|=n-j}\left\{C_{s, 1}^{k}\right\}<R_{1}^{s}, \max _{p \in \Psi,|\Psi|=j}\left\{C_{s, 2}^{p}\right\}<R_{2}^{s}\right\} \\
& =\sum_{j=0}^{n} C_{n}^{j} \operatorname{Pr}\left\{\min _{i \in \Psi,|\Psi|=j}\left\{C_{s, 1}^{i}\right\}>R_{1}^{s}, \max _{p \in \Psi,|\Psi|=j}\left\{C_{s, 2}^{p}\right\}<R_{2}^{s}\right\} \operatorname{Pr}\left\{\max _{k \in \bar{\Psi},|\bar{\Psi}|=n-j}\left\{C_{s, 1}^{k}\right\}<R_{1}^{s}\right\} \\
& =\sum_{j=0}^{n} C_{n}^{j}(\underbrace{\operatorname{Pr}\left\{C_{s, 1}^{i}>R_{1}^{s}, C_{s, 2}^{i}<R_{2}^{s}\right\}}_{\triangleq})^{j}(\underbrace{\operatorname{Pr}\left\{C_{s, 1}^{k}<R_{1}^{s}\right\}}_{\triangleq_{\Delta_{3}}})^{n} \\
& =\left(\Delta_{2}+\Delta_{3}\right)^{n} \\
& =\left(\operatorname{Pr}\left\{C_{s, 1}^{i}>R_{1}^{s}, C_{s, 2}^{i}<R_{2}^{s}\right\}+\operatorname{Pr}\left\{C_{s, 1}^{i}<R_{1}^{s}\right\}\right)^{n} \\
& =\left(\operatorname{Pr}\left\{C_{s, 1}^{i}<R_{1}^{s} \text { or } C_{s, 2}^{i}<R_{2}^{s}\right\}\right)^{n} \\
& =\left(1-\operatorname{Pr}\left\{C_{s, 1}^{i}>R_{1}^{s}, C_{s, 2}^{i}>R_{2}^{s}\right\}\right)^{n}
\end{aligned}
$$

where $i=1,2, \rho_{3}=\frac{\left(1-\alpha_{\mathrm{J}}\right) P_{R}}{\sigma^{2}}, \rho_{4}=\frac{\alpha_{\mathrm{J}} P_{R}}{\sigma^{2}}$, and $H_{E}=$ $\max _{k \in \bar{\Phi}}\left\{G_{E}^{k}\right\}$.

We assume that both users are aware of the jamming signals, which means jamming signals do not influence the SINR at both users [28], [32], [33], [34]. It must be noted that the special case of $n=K$ or $\alpha_{\mathrm{J}}=0$, ODRS scheme reduces to OSRS scheme. Then the SOP with ODRS scheme is expressed as

$$
P_{\text {out }}^{\text {ODRS }}=\sum_{n=0}^{K-1} \operatorname{Pr}(|\Phi|=n) P_{\Phi_{n}}^{\mathrm{J}}+P_{\Phi_{K}}^{\mathrm{OSRS}},
$$

where $P_{\Phi_{K}}^{\text {OSRS }}=\left(1-\Delta_{1}\right)^{K}$.

Similar to 27, the SOP of the cooperative NOMA system on condition that $|\Phi|=n(n<K)$ can be expressed as 31, given at the top of this page, where $\ell=\frac{\theta_{1}-1}{\alpha_{1} \rho_{3}}, w=-\frac{1}{\alpha_{1} \rho_{3}}$, $u=\frac{\alpha_{2}}{\alpha_{1} \theta_{2}-1}, v=\frac{\alpha_{1} \alpha_{2} \theta_{2} \rho_{3}}{1-a_{1} \theta_{2}}$, and $Y=\frac{G_{E}^{k}}{1+\rho_{4} H_{E}}$.

To achieve the analytical expression of $\Delta_{4}$, the PDF of $H_{E}$ and $Y$ are given in Lemma $\mathbf{1}$ and Lemma 2, respectively.

Lemma 1: Given as $|\bar{\Phi}|=K-n(n<K)$, the PDF of $H_{E}$ is given by

$$
f_{H_{E}}(z)=\frac{(K-n) \lambda_{E}^{m_{E}}}{\Gamma\left(m_{E}\right)} \sum_{S_{E}} A z^{B+m_{E}-1} e^{-C \lambda_{E} z}
$$

where $S_{E}=\left\{\left(n_{1}, \cdots, n_{m_{E}+1}\right) \in \mathbb{N}\left|\sum_{p=1}^{m_{E}+1} n_{p}=K-n-1\right|\right\}$,
$A=\left(\frac{(K-n-1) !}{\frac{\left(K-m_{E}^{+1}\right)}{\prod_{q=1}\left(n_{q}\right) !}} \prod_{p=2}^{m_{E}+1}\left(-\frac{\lambda_{E}^{p-2}}{(p-2) !}\right)^{n_{p}}, \quad B=\right.$ $\sum_{p=2}^{m_{E}+1} n_{p}(p-2)$, and $C=1+\sum_{p=2}^{m_{E}+1} n_{p}$.

Proof : See Appendix A.

Lemma 2: The PDF of $Y$ is given by

$$
\begin{aligned}
f_{Y}(y)= & \varphi_{0} \sum_{k=0}^{m_{E}-1} \sum_{S_{E}} \sum_{j=0}^{k} \frac{\delta e^{-\lambda_{E} y}}{\left(\rho_{4} y+C\right)^{\varsigma+1}} \\
& \times\left(\rho_{4} \lambda_{E} y^{k+1}+D y^{k}-C k y^{k-1}\right),
\end{aligned}
$$

where $\varphi_{0}=\frac{(K-n) \lambda_{E}^{m_{E}}}{\Gamma\left(m_{E}\right)}, \varsigma=B+m_{E}+j, \delta=$ $\frac{C_{k}^{j} A \rho_{4}^{j} \lambda_{E}^{k-\varsigma}(\varsigma-1) !}{k !}$, and $D=C \lambda_{E}-\rho_{4} k+\rho_{4} \varsigma$.

Proof : See Appendix B.

Similar to (24), based on (12) and (13), we obtain the analytical expression of $\Delta_{4}$ as $(34)$, given at the top of the next page, where $\Xi_{3}=w^{q} h\left(\frac{1}{v}, p, q, \lambda_{1} \theta_{1}+\lambda_{E}, \lambda_{2} w u, u, v, \ell\right)$ and

$$
\begin{aligned}
h(a, b, c, f, r, u, v, \ell) & =\int_{0}^{a}\left(\ell+\theta_{1} y\right)^{b}\left(1+\frac{u}{1-v y}\right)^{c} \\
& \times \frac{\left(\rho_{4} \lambda_{E} y^{k+1}+D y^{k}-C k y^{k-1}\right)}{\left(\rho_{4} y+C\right)^{\varsigma+1} e^{f y+\frac{r}{1-v y}}} d y .
\end{aligned}
$$

Similar to 17, utilizing Gaussian-Chebyshev quadrature, 


$$
\begin{aligned}
\Delta_{4} & =\int_{0}^{\frac{1}{v}} \operatorname{Pr}\left\{G_{1}^{k}>\ell+\theta_{1} y, G_{2}^{k}>w+\frac{w u}{1-v y}\right\} f_{Y}(y) d y \\
& =\int_{0}^{\frac{1}{v}}\left(1-F_{G_{1}^{k}}\left(\ell+\theta_{1} y\right)\right)\left(1-F_{G_{2}^{k}}\left(w+\frac{w u}{1-v y}\right)\right) f_{Y}(y) d y \\
& =e^{-\lambda_{1} \ell-\lambda_{2} w} \varphi_{0} \sum_{p=0}^{m_{U}-1} \sum_{q=0}^{m_{U}-1} \sum_{k=0}^{m_{E}-1} \sum_{S_{E}} \sum_{j=0}^{k} \frac{\delta \lambda_{1}^{p} \lambda_{2}^{q} \Xi_{3}}{p ! q !}
\end{aligned}
$$

$$
\begin{aligned}
P_{\Phi_{n}}^{\mathrm{TMRF}, \infty} & =1-F_{G_{E}^{\mathrm{TMRF}}}\left(a_{1}\right)+\varphi_{1} \beta_{E}^{\mathrm{TMRF}} \sum_{k=0}^{\tau_{U}} \frac{C_{\tau_{U}}^{k} \theta_{1}^{k} b_{1}^{\tau_{U}-k} \Upsilon\left(k+\tau_{E}, \lambda_{E} a_{1}\right)}{\lambda_{E}^{k+\tau_{E}}} \\
& +\varphi_{2} \beta_{E}^{\mathrm{TMRF}} c_{1}^{\tau_{U}} g\left(a_{1}, \tau_{E}, 0, \frac{\alpha_{2}}{c_{1} d_{1}}, \frac{e_{1}}{d_{1}}, \lambda_{E}, 0,0, \tau_{U}\right) \\
& -\varphi_{1} \varphi_{2} \beta_{E}^{\mathrm{TMRF}} b_{1}^{\tau_{U}} c_{1}^{\tau_{U}} g\left(a_{1}, \tau_{E}, \frac{\theta_{1}}{b_{1}}, \frac{\alpha_{2}}{c_{1} d_{1}}, \frac{e_{1}}{d_{1}}, \lambda_{E}, 0, \tau_{U}, \tau_{U}\right) \stackrel{(a)}{\approx} 1-F_{G_{E}^{\mathrm{TMRF}}}\left(a_{1}\right)
\end{aligned}
$$

we have

$$
\begin{aligned}
h(a, b, c, f, r, u, v, \ell) & \approx \frac{a}{2} \sum_{i=1}^{N} w_{i}\left(\ell+\theta_{1} s_{j}\right)^{b}\left(1+\frac{u}{1-v s_{j}}\right)^{c} \\
& \times \frac{\left(\rho_{4} \lambda_{E} s_{j}^{k+1}+D s_{j}^{k}-C k s_{j}^{k-1}\right)}{\left(\rho_{4} s_{j}+C\right)^{\varsigma+1} e^{f s_{j}+\frac{r}{1-v s_{j}}}},
\end{aligned}
$$

where $s_{j}=\frac{a}{2}\left(t_{i}+1\right)$.

It must be noted that only one relay is selected in both OSRS and TSRS schemes while two relays are selected in ODRS scheme in which the second relay is selected from those that can not decode mixed signals from $S$ to transmit jamming signals to fight against the eavesdropper.

Remark 5: The results in [29] and [30] showed that increasing the transmit power will improve the secrecy performance of the NOMA systems until the secrecy outage performance reaches a ceiling. Then we can easily draw a conclusion that the ODRS scheme can obtain better secrecy performance than TMRF and OSRS schemes in higher- $\rho_{R}$ region because the jamming signals apparently decrease the SINR of the eavesdroppers. The same results are for those scenarios when the main links have better channel quality. These conclusions are verified by Figs. 2 - 6 and Figs. 9 - 13 , respectively.

\section{Asymptotic Secrecy Outage Probability ANALYSIS}

To obtain more insights, in this section we analyze the asymptotic SOP in the higher SINR regime. Similar to [29] and [30], we assume that $\Omega_{2} \rightarrow \infty, \Omega_{1}=\varepsilon_{1} \Omega_{2}\left(\varepsilon_{1}>1\right) 4$ and $\Omega_{R}=\varepsilon_{2} \Omega_{2}$, where $\varepsilon_{1}$ and $\varepsilon_{2}$ are constant. The asymptotic SOP is expressed as [51]

$$
P_{\mathrm{out}}^{\infty}=\left(G_{a} \Omega_{2}\right)^{-G_{d}}+\mathcal{O}\left(\Omega_{2}^{-G_{d}}\right)
$$

where $G_{a}$ and $G_{d}$ denotes the SDO and SAG, which characterize the slope and the SNR advantage of $P_{\text {out }}^{\infty}$ compared with the reference curve $\Omega_{2}^{-G_{d}}$, respectively.

\footnotetext{
${ }^{4}$ Since $U_{1}$ has better channel condition than $U_{2}$, then we assume $\varepsilon_{1}>1$. Since there is no similar relationship between the $\Omega_{R}$ and $\Omega_{1}$, thus there is no condition on $\varepsilon_{2}$.
}

The asymptotic CDF of $G_{v}^{k}(v \in\{1,2\})$ can be expressed as [51]

$$
F_{G_{v}^{k}}^{\infty}(x)=\varphi_{v} x^{\tau_{U}}+\mathcal{O}\left(x^{\tau_{U}}\right),
$$

where $\varphi_{1}=\frac{m_{U}^{\tau_{U}}}{\left(\varepsilon_{1} \Omega_{2}\right)^{\tau_{U}}\left(\tau_{U}\right) !}, \varphi_{2}=\frac{m_{U}^{\tau_{U}}}{\Omega_{2}^{\tau_{U}}\left(\tau_{U}\right) !}$, and $\mathcal{O}(\cdot)$ denotes higher order terms. Then the asymptotic SOP of cooperative NOMA systems is obtained as

$$
P_{\mathrm{out}}^{\infty}=\sum_{n=0}^{K} C_{K}^{n} \varphi_{R}^{K-n} \eta^{m_{R}(K-n)} P_{\Phi_{n}}^{\infty},
$$

where $\varphi_{R}=\frac{m_{R} m_{R}}{\left(\varepsilon_{2} \Omega_{2}\right)^{m} R\left(m_{R}\right) !}$. The $P_{\Phi_{n}}^{\infty}$ for all the RS schemes is given as follows.

\section{A. Traditional Multiple Relay Forwarding Scheme}

Based on (15), we have the analytical for SOP with TMRF scheme as 39p, given at the top of this page, where $\Upsilon(\cdot, \cdot)$ is the lower incomplete Gamma function, as defined by $(8.350 .1)$ of [50]. The (a) follows from $\varphi_{i} \rightarrow 0(i \in\{1,2\})$ with $\Omega_{2} \rightarrow$ $\infty$.

Remark 6: Based on 97 and (39), we have $G_{d}^{\mathrm{TMRF}}=0$ because it is independent of $\Omega_{2}$.

\section{B. Optimal Single Relay Selection Scheme}

Based on 23, we obtain

$$
P_{\Phi_{n}}^{\mathrm{OSRS}, \infty}=\left(1-\Delta_{1}^{\infty}\right)^{n},
$$

where $\Delta_{1}^{\infty}$ is given in (41) at the top of the next page, where $\varphi_{3}=\frac{m_{U}^{m_{U}}}{\left(\varepsilon_{1} \Omega_{2}\right)^{m_{U}}\left(m_{U}\right) !}$ and $\varphi_{4}=\frac{m_{U}^{m_{U}}}{\Omega_{2}^{m_{U}}\left(m_{U}\right) !}$. The (b) follows from $\varphi_{j} \rightarrow 0(j \in\{3,4\})$ with $\Omega_{2} \rightarrow \infty$.

\section{Optimal Dual Relay Selection Scheme}

Based on 30, we obtain the asymptotic SOP for cooperative NOMA systems with ODRS scheme as

$$
P_{\text {out }}^{\mathrm{ODRS}, \infty}=\sum_{n=0}^{K-1} C_{K}^{n} \varphi_{R}^{K-n} \eta^{m_{R}(K-n)} P_{\Phi_{n}}^{\mathrm{ODRS}, \infty}+P_{\Phi_{K}}^{\mathrm{OSRS}, \infty},
$$




$$
\begin{aligned}
\Delta_{1}^{\infty} & =F_{G_{E}^{m}}\left(a_{2}\right)-\varphi_{3} \beta_{E} \sum_{k=0}^{m_{U}} \frac{C_{m_{U}}^{k} \theta_{1}^{k} b_{2}^{m_{U}-k} \Upsilon\left(k+m_{E}, \lambda_{E} a_{2}\right)}{\lambda_{E}^{k+m_{E}}}-\varphi_{4} \beta_{E} c_{2}^{m_{U}} g\left(a_{2}, m_{E}, 0, \frac{\alpha_{2}}{c_{2} d_{2}}, \frac{e_{2}}{d_{2}}, \lambda_{E}, 0,0, m_{U}\right) \\
& +\varphi_{3} \varphi_{4} \beta_{E} b_{2}^{m_{U}} c_{2}^{m_{U}} g\left(a_{2}, m_{E}, \frac{\theta_{1}}{b_{2}}, \frac{\alpha_{2}}{c_{2} d_{2}}, \frac{e_{2}}{d_{2}}, \lambda_{E}, 0, m_{U}, m_{U}\right) \stackrel{(b)}{\approx} F_{G_{E}^{m}}\left(a_{2}\right)
\end{aligned}
$$

$$
\begin{aligned}
\Delta_{4}^{\infty} & =1-\varphi_{0} \sum_{k=0}^{m_{E}-1} \sum_{S_{E}} \sum_{j=0}^{k} \frac{\delta e^{-\frac{\lambda_{E}}{v}}}{\left(C+\frac{\rho_{4}}{v}\right)^{\varsigma} v^{k}}-\varphi_{0} \varphi_{3} \sum_{k=0}^{m_{E}-1} \sum_{S_{E}} \sum_{j=0}^{k} \delta h\left(\frac{1}{v}, m_{U}, 0, \lambda_{E}, 0, u, v, \ell\right) \\
& -\varphi_{0} \varphi_{4} w^{m_{U}} \sum_{k=0}^{m_{E}-1} \sum_{S_{E}} \sum_{j=0}^{k} \delta h\left(\frac{1}{v}, 0, m_{U}, \lambda_{E}, 0, u, v, \ell\right)+\varphi_{0} \varphi_{3} \varphi_{4} w^{m_{U}} \sum_{k=0}^{m_{E}-1} \sum_{S_{E}} \sum_{j=0}^{k} \delta h\left(\frac{1}{v}, m_{U}, m_{U}, \lambda_{E}, 0, u, v, \ell\right) \\
& \stackrel{(c)}{\approx} 1-\varphi_{0} \sum_{k=0}^{m_{E}-1} \sum_{S_{E}} \sum_{j=0}^{k} \frac{\delta e^{-\frac{\lambda_{E}}{v}}}{\left(C+\frac{\rho_{4}}{v}\right)^{\varsigma} v^{k}}
\end{aligned}
$$

where $P_{\Phi_{K}}^{\mathrm{OSRS}, \infty}=\left(1-\Delta_{1}^{\infty}\right)^{K}$. Based on 31, we have

$$
P_{\Phi_{n}}^{\text {ODRS, } \infty}=\left(1-\Delta_{4}^{\infty}\right)^{n},
$$

where $\Delta_{4}^{\infty}$ was shown in 444, shown at the top of this page. The (c) follows from $\varphi_{j} \rightarrow 0(j \in\{3,4\})$ with $\Omega_{2} \rightarrow \infty$.

Remark 7: Based on the definition of SDO as $G_{d}=$ $-\lim _{\Omega_{2} \rightarrow \infty} \frac{\ln \left(P_{\text {out }}^{\infty}\right)}{\ln \left(\Omega_{2}\right)}$, we obtain $G_{d}^{\mathrm{TMRF}}=G_{d}^{\mathrm{OSRS}}=G_{d}^{\mathrm{ODRS}}=0$ because the first term is independent of $\Omega_{2}$ in 41) and (44, , respectively.

Remark 8: Based on (4), one can observe that with main channel gains increasing, the received SINR at $U_{2}$ has an upper bound, which is a constant $\frac{\alpha_{2}}{\alpha_{1}}$. Thus, the part $\operatorname{Pr}\left\{C_{s, 2}^{\mathrm{TMRF}}<R_{2}^{s}\right\}$ in 15 will be a constant when $\Omega_{2} \rightarrow \infty$. This is the reason that the SDO of cooperative NOMA with FPA scheme is zero. One can obtain the same result for the part $\operatorname{Pr}\left\{G_{2}^{m} \geq \delta_{4}\left(G_{E}^{m}\right)\right\}$ in 23 . The results were testified in [29] and [30] that the SDO of NOMA systems is determined by the far user. This is the reason that the SDO of cooperative NOMA with FPA scheme is zero.

\section{Asymptotic Secrecy Outage Probability} Analysis With Dynamic Power Allocation Scheme

Since there is a ceiling for the SINR of $U_{2}$, which equals to $\frac{\alpha_{2}}{\alpha_{1}}$, there is a floor for the SOP of NOMA systems when the transmit SNR increases. A new DPA scheme was proposed in [29] and the PA coefficients are given as $\alpha_{1}^{\mathrm{DPA}}=\frac{1}{1+\mu \lambda_{2}^{-\varpi}}$ and $\alpha_{2}^{\mathrm{DPA}}=\frac{\mu \lambda_{2}^{-\varpi}}{1+\mu \lambda_{2}^{-\varpi}}$, where $0<\varpi<1$ and $\mu>1$. In this section, the asymptotic SOP is analyzed while this DPA scheme is utilized.

\section{A. Traditional Multiple Relay Forwarding Scheme with Dy- namic Power Allocation Scheme}

We achieve the asymptotic SOP under TMRF-DPA scheme as (45), shown at the top of the next page. Then the SDO of the cooperative NOMA systems in this case is obtained as

$$
G_{d}^{\mathrm{TMRF}, \mathrm{DPA}}=K \min \left\{m_{U}(1-\varpi), m_{R}\right\},
$$

and the SAG in this case is given in (47), shown at the top of the next page.

Proof : See Appendix C.

\section{B. Optimal Single Relay Selection Scheme with Dynamic} Power Allocation Scheme

Similar to 40], we have

$$
P_{\Phi_{n}}^{\mathrm{OSRS}, \infty, \mathrm{DPA}}=\left(1-\Delta_{1}^{\infty, \mathrm{DPA}}\right)^{n}
$$

where $\Delta_{1}^{\infty, \mathrm{DPA}}$ is given by 49 , shown at the top of the next page. Similar to (46) and 477, we obtain the SDO of the cooperative NOMA systems in this case as

$$
\begin{aligned}
G_{d}^{\mathrm{OSRS}, \mathrm{DPA}} & =\min _{0 \leq n \leq K}\left\{m_{R}(K-n)+n m_{U}(1-\varpi)\right\} \\
& =G_{d}^{\mathrm{TMRF}, \mathrm{DPA}},
\end{aligned}
$$

and the SAG is given in (51), shown at the top of the next page.

Remark 9: Based on (46) and (50), one can observe the SDO of cooperative NOMA systems under both TMRF-DPA and OSRS-DPA schemes equals to the minimum SDO of dual hops. Moreover, it is a full diversity order based on the results in [29] and [30].

\section{Optimal Dual Relay Selection with Dynamic Power Allo- cation Scheme}

Similar to (42), we write the asymptotic SOP for the cooperative NOMA system under ODRS-DPA scheme as

$$
\begin{aligned}
P_{\mathrm{out}}^{\mathrm{ODRS}, \infty, \mathrm{DPA}} & =\sum_{n=0}^{K-1} C_{K}^{n} \varphi_{R}^{K-n} \eta^{m_{R}(K-n)} P_{\Phi_{n}}^{\mathrm{ODRS}, \infty, \mathrm{DPA}} \\
& +P_{\Phi_{K}}^{\mathrm{OSRS}, \infty, \mathrm{DPA}},
\end{aligned}
$$

where $P_{\Phi_{K}}^{\mathrm{OSRS}, \infty, \mathrm{DPA}}=\left(1-\Delta_{1}^{\infty, \mathrm{DPA}}\right)^{K}$. Similar to 43 , we have

$$
P_{\Phi_{n}}^{\mathrm{ODRS}, \infty, \mathrm{DPA}}=\left(1-\Delta_{4}^{\infty, \mathrm{DPA}}\right)^{n}
$$




$$
\begin{aligned}
& P_{\Phi_{n}}^{\mathrm{TMRF}, \infty, \mathrm{DPA}}=\underbrace{1-F_{G_{E}^{\mathrm{TMRF}}}\left(a_{1}^{\mathrm{DPA}}\right)}_{I_{1}^{\mathrm{TMRF}}}+\underbrace{\varphi_{1} \beta_{E}^{\mathrm{TMRF}} \sum_{k=0}^{\tau_{U}} \frac{C_{\tau_{U}}^{k} \theta_{1}^{k}\left(b_{1}^{\mathrm{DPA}}\right)^{\tau_{U}-k} \Upsilon\left(k+\tau_{E}, \lambda_{E} a_{1}^{\mathrm{DPA}}\right)}{\lambda_{E}^{k+\tau_{E}}}}_{I_{2}^{\mathrm{TMRF}}} \\
& +\underbrace{\varphi_{2} \beta_{E}^{\mathrm{TMRF}}\left(c_{1}^{\mathrm{DPA}}\right)^{\tau_{U}} g\left(a_{1}^{\mathrm{DPA}}, \tau_{E}, 0, \frac{\alpha_{2}^{\mathrm{DPA}}}{c_{1}^{\mathrm{DPA}} d_{1}^{\mathrm{DPA}}}, \frac{e_{1}^{\mathrm{DPA}}}{d_{1}^{\mathrm{DPA}}}, \lambda_{E}, 0,0, \tau_{U}\right)}_{I_{3}^{\text {TMRF }}} \\
& -\underbrace{\varphi_{1} \varphi_{2} \beta_{E}^{\mathrm{TMRF}}\left(b_{1}^{\mathrm{DPA}}\right)^{\tau_{U}}\left(c_{1}^{\mathrm{DPA}}\right)^{\tau_{U}} g\left(a_{1}^{\mathrm{DPA}}, \tau_{E}, \frac{\theta_{1}}{b_{1}^{\mathrm{DPA}}}, \frac{\alpha_{2}^{\mathrm{DPA}}}{c_{1}^{\mathrm{DPA}} d_{1}^{\mathrm{DPA}}}, \frac{e_{1}^{\mathrm{DPA}}}{d_{1}^{\mathrm{DPA}}}, \lambda_{E}, 0, \tau_{U}, \tau_{U}\right)}_{I_{4}^{\mathrm{TMRF}}} \stackrel{(d)}{\approx} I_{2}^{\mathrm{TMRF}}
\end{aligned}
$$

$$
G_{a}^{\mathrm{TMRF}, \mathrm{DPA}}=\left\{\begin{array}{cl}
\frac{1}{m_{U}}\left(\frac{\varepsilon_{1} \rho_{1}}{\mu\left(\theta_{1}-1\right)}\right)^{\frac{1}{1-\varpi}}\left(\left(K m_{U}\right) !\right)^{\frac{1}{K m_{U}(1-\varpi)}}, & m_{R}>m_{U}(1-\varpi) \\
\frac{\varepsilon_{2}\left(\left(m_{R}\right) !\right)^{\frac{1}{m_{R}}}}{\eta m_{R}}, & m_{R}<m_{U}(1-\varpi)
\end{array}\right.
$$

$$
\begin{aligned}
\Delta_{1}^{\infty, \mathrm{DPA}} & =\underbrace{F_{E}\left(a_{2}^{\mathrm{DPA}}\right)}_{I_{1}^{\mathrm{OSRS}}}-\underbrace{\varphi_{3} \beta_{E} \sum_{k=0}^{m_{U}} \frac{C_{m_{U}}^{k} \theta_{1}^{k}\left(b_{2}^{\mathrm{DPA}}\right)^{m_{U}-k} \Upsilon\left(k+m_{E}, \lambda_{E} a_{2}^{\mathrm{DPA}}\right)}{\lambda_{E}^{k+m_{E}}}}_{I_{2}^{\mathrm{OSRS}}} \\
& -\underbrace{\varphi_{4} \beta_{E}\left(c_{2}^{\mathrm{DPA}}\right)^{m_{U}} g\left(a_{2}^{\mathrm{DPA}}, m_{E}, 0, \frac{\alpha_{2}^{\mathrm{DPA}}}{\left.c_{2}^{\mathrm{DPA}} d_{2}^{\mathrm{DPA}}, \frac{e_{2}^{\mathrm{DPA}}}{d_{2}^{\mathrm{DPA}}}, \lambda_{E}, 0,0, m_{U}\right)}\right.}_{I_{3}^{\mathrm{OSRS}}} \\
& +\underbrace{\varphi_{3} \varphi_{4} \beta_{E}\left(b_{2}^{\mathrm{DPA}}\right)^{m_{U}}\left(c_{2}^{\mathrm{DPA}}\right)^{m_{U}} g\left(a_{2}^{\mathrm{DPA}}, m_{E}, \frac{\theta_{1}}{b_{2}^{\mathrm{DPA}}}, \frac{\alpha_{2}^{\mathrm{DPA}}}{c_{2}^{\mathrm{DPA}} d_{2}^{\mathrm{DPA}}}, \frac{e_{2}^{\mathrm{DPA}}}{d_{2}^{\mathrm{DPA}}}, \lambda_{E}, 0, m_{U}, m_{U}\right)}_{I_{4}^{\mathrm{OSRS}}} \stackrel{(d)}{\approx} 1-I_{2}^{\mathrm{OSRS}}
\end{aligned}
$$

$$
G_{a}^{\mathrm{OSRS}, \mathrm{DPA}}=\left\{\begin{array}{cl}
\frac{1}{m_{U}}\left(\frac{\varepsilon_{1} \rho_{2}}{\mu\left(\theta_{1}-1\right)}\right)^{\frac{1}{1-\varpi}}\left(\left(m_{U}\right) !\right)^{\frac{1}{m_{U}(1-\varpi)}}, & m_{R}>m_{U}(1-\varpi) \\
\frac{\varepsilon_{2}\left(m_{R} !\right)^{\frac{1}{m_{R}}}}{\eta m_{R}}, & m_{R}<m_{U}(1-\varpi)
\end{array}\right.
$$

where $\Delta_{4}^{\infty, \mathrm{DPA}}$ is shown in 54 at the top of the next page, where $w^{\mathrm{DPA}}=-\frac{1}{\alpha_{1}^{\mathrm{DPA}} \rho_{3}}, v^{\mathrm{DPA}}=\frac{\alpha_{1}^{\mathrm{DPA}} \alpha_{2}^{\mathrm{DPA}} \theta_{2} \rho_{3}}{1-\alpha_{1}^{\mathrm{DPA}} \theta_{2}}, u^{\mathrm{DPA}}=$ $\frac{\alpha_{2}^{\mathrm{DPA}}}{\alpha_{1}^{\mathrm{DPA}} \theta_{2}-1}$, and $\ell^{\mathrm{DPA}}=\frac{\theta_{1}-1}{\alpha_{1}^{\mathrm{DPA}} \rho_{3}}$.

Similar to 46 and 477, we obtain the SDO and SAG of the cooperative NOMA systems in this case as (55) and (56) at the top of the next page, respectively, where $H_{1}=m_{U}(1-\varpi)-\varpi$, $H_{2}=m_{U}(1-\varpi)+\varpi(K-1)$, and $H_{3}=$ $\sum_{k=0}^{m_{E}-1} \sum_{S_{E}} \sum_{j=0}^{k} \frac{\delta \mu^{m_{U}+1}}{2 \theta_{2} \rho_{3}}\left(\frac{\theta_{1}-1}{\rho_{3}}\right)^{m_{U}} \sum_{i=1}^{N} \frac{w_{i}\left(\rho_{4} \lambda_{E} s_{j}^{k+1}+D s_{j}^{k}-C k s_{j}^{k-1}\right)}{\left(\rho_{4} s_{j}+C\right)^{\varsigma+1} e^{\lambda_{E} s_{j}}}$ $\frac{\varphi_{0} m_{U}^{m_{U}}(1-\varpi)-w}{\varepsilon_{1}^{m}\left(m_{U}\right) !}$.

Remark 10: It was testified that non-zero diversity order can be obtained due to $\frac{\alpha_{2}^{\mathrm{DA}}}{\alpha_{1}^{\mathrm{DPA}}}=\mu \lambda_{2}^{-\varpi} \rightarrow \infty$ with $\Omega_{2} \rightarrow \infty$ in [29] and [30]. Based on (46), (50), and (55), one can find that $\varpi$ exhibits a strong influence on the SDO of the cooperative NOMA systems. The SDO for cooperative NOMA systems under TMRF-DPA and OSRS-DPA will reduce as $\varpi$ increases and there is a ceiling as $K m_{R}$, which is proved by Figs. 14 and 15 of this work.

Remark 11: Based on 55, one can realize that using a specific relay to send jamming signals may reduce the SDO of cooperative NOMA systems in some conditions.

\section{COMPARISON AND DISCUSSION}

To identify the strengths and weakness of each proposed scheme, a more detailed secrecy outage performance comparison for all the proposed schemes is summarized in Table [1] which is at the top of the next page. In general, the realization of all the schemes depends heavily on the available CSI of all the links at the source. It is worth noting that the CSI of the first hop is essential in all proposed schemes to distinguish between available and unavailable relays.

The main difference among all the proposed schemes is whether the CSI of the second hop is available or not. For the TMRF, there is no selection and the MRC is utilized at all 


$$
\begin{aligned}
& \Delta_{4}^{\infty, \mathrm{DPA}}=1-\underbrace{\varphi_{0} \sum_{k=0}^{m_{E}-1} \sum_{S_{E}} \sum_{j=0}^{k} \delta e^{-\frac{\lambda_{E}}{v^{\mathrm{DPA}}}\left(C+\frac{\rho_{4}}{v^{\mathrm{DPA}}}\right)^{-\varsigma}\left(v^{\mathrm{DPA}}\right)^{-k}}}_{I_{1}^{\mathrm{ODRS}}} \\
& -\underbrace{\varphi_{0} \varphi_{3} \sum_{k=0}^{m_{E}-1} \sum_{S_{E}} \sum_{j=0}^{k} \delta h\left(\frac{1}{\left.v^{\mathrm{DPA}}, m_{U}, 0, \lambda_{E}, 0, u^{\mathrm{DPA}}, v^{\mathrm{DPA}}, \ell^{\mathrm{DPA}}\right)}\right.}_{I_{2}^{\mathrm{ODRS}}} \\
& -\underbrace{\varphi_{0} \varphi_{4}\left(w^{\mathrm{DPA}}\right)^{m_{U}} \sum_{k=0}^{m_{E}-1} \sum_{S_{E}} \sum_{j=0}^{k} \delta h\left(\frac{1}{v^{\mathrm{DPA}}}, 0, m_{U}, \lambda_{E}, 0, u^{\mathrm{DPA}}, v^{\mathrm{DPA}}, \ell^{\mathrm{DPA}}\right)}_{I_{3}^{\text {ODRS }}}
\end{aligned}
$$

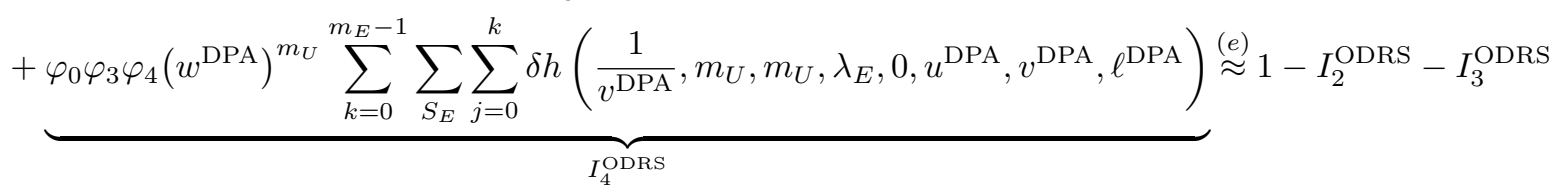

$$
\begin{aligned}
G_{d}^{\text {ODRS,DPA }} & =\min \left\{\min _{0 \leq n \leq K-1}\left\{m_{R}(K-n)+n H_{1}\right\}, K(1-\varpi) m_{U}\right\} \\
& =\min \left\{K m_{R},(K-1)\left(m_{U}(1-\varpi)-\varpi\right)+m_{R}, K m_{U}(1-\varpi)\right\}
\end{aligned}
$$

$$
G_{a}^{\text {ODRS,DPA }}= \begin{cases}\frac{\varepsilon_{2}\left(m_{R} !\right)^{\frac{1}{m_{R}}}}{\eta m_{R}}, & m_{R}<H_{1} \\ \left(\frac{K\left(\eta m_{R}\right)^{m_{R}} H_{3}^{K-1}}{\varepsilon_{2}^{m_{R}}\left(m_{R}\right) !}\right)^{-\frac{1}{(K-1) H_{1}+m_{R}}}, & H_{1}<m_{R}<H_{2} \\ \frac{1}{m_{U}}\left(\left(m_{U}\right) !\right)^{\frac{1}{m_{U}(1-\varpi)}}\left(\frac{\varepsilon_{1} \rho_{2}}{\mu\left(\theta_{1}-1\right)}\right)^{\frac{1}{1-\varpi}}, & m_{R}>H_{2}\end{cases}
$$

TABLE I

Comparison of the Proposed Schemes

\begin{tabular}{c|c|c|c|c|c|c}
\hline Scheme & $\begin{array}{c}\text { CSI Requirement } \\
\text { at 2nd hop }\end{array}$ & Criterion & SDO & SAG & $\begin{array}{c}\text { Secrecy } \\
\text { Performance }\end{array}$ & Complexity \\
\hline TMRF & - & - & Eq. (47) & Eq. (48) & Worst & Low \\
\hline OSRS/TSRS & $\begin{array}{c}R_{j \in \Phi}-U, \\
R_{j \in \Phi}-E\end{array}$ & Eq. (22)/(26) & Eq. (51) & Eq. (52) & Second Best & Middle \\
\hline ODRS & $\begin{array}{c}R_{i \in \Re}-U, \\
R_{i \in \Re}-E\end{array}$ & Eq. (22), (28) & Eq. (56) & Eq. (57) & Best & High \\
\hline
\end{tabular}

the destinations, thus the CSI of second hop is not required. For the OSRS/TSRS, the forwarding relay is selected based on the normalized secrecy outage performance. Thus, the CSI between all the available relays (those that can correctly decode mixed signals from the source) and the destinations (all the users and the eavesdropper) is necessary. For the ODRS, all the CSI of the second hop is essential since an additional jamming relay is selected besides selection of the forwarding relay. As we all know, CSI is estimated at the destination that is fed back to the source node over some proper control channels. More sophisticated selection process need more CSI. Difference in CSI requirement causes the difference in overhead for control channels.
One can observe that the more CSI available, the better the secrecy outage performance will be obtained. We can easily find that the TSRS has the same secrecy outage performance to the OSRS, which outperforms that of TMRF. Furthermore, the ODRS can obtain better secrecy performance than that of OSRS at the cost of higher complexity.

\section{NUMERICAL RESUlTS AND DisCUSSIONS}

In this section, we present numerical results and MonteCarlo simulations to testify our analysis. The main adopted parameters are set to $\rho_{S}=\rho_{R}=\rho, R_{1}^{\text {th }}=0.2$ nat per channel use, $R_{2}^{\text {th }}=0.1$ nat per channel use, and $\sigma^{2}=1$. The GaussianChebyshev parameter is chosen as $N=30$. In all the figures, 


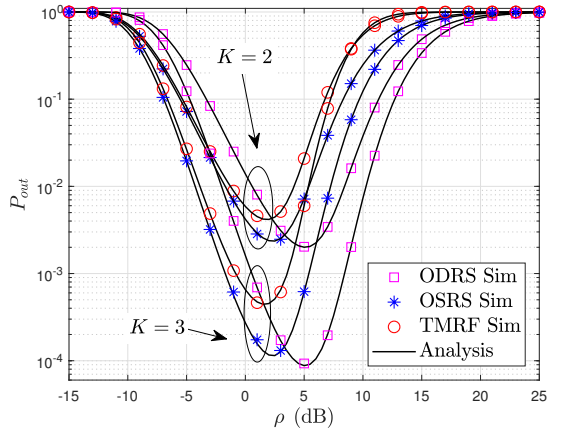

Fig. 2. SOP for various $K$ with $R_{1}=0.1, R_{2}=0.2, m_{U}=m_{E}=m_{R}=$ $2, \alpha_{\mathrm{J}}=0.5, \Omega_{1}=12 \mathrm{~dB}, \Omega_{R}=\Omega_{2}=10 \mathrm{~dB}$, and $\Omega_{E}=-5 \mathrm{~dB}$.

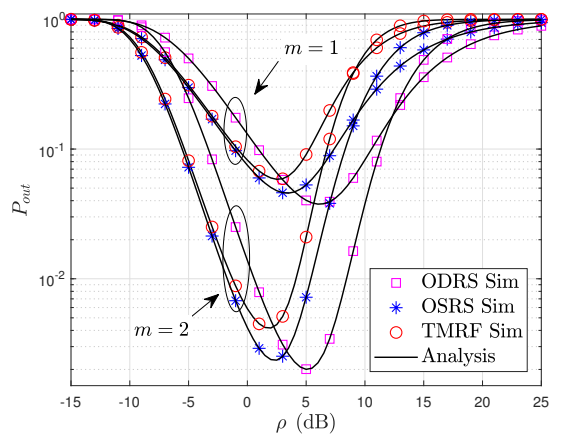

Fig. 3. SOP for various $m$ with $R_{1}=0.1, R_{2}=0.2, m_{U}=m_{E}=m_{R}=$ $m, \alpha_{1}=0.2, \alpha_{\mathrm{J}}=0.5, \Omega_{1}=12 \mathrm{~dB}, \Omega_{R}=\Omega_{2}=10 \mathrm{~dB}$, and $\Omega_{E}=-5$ dB.

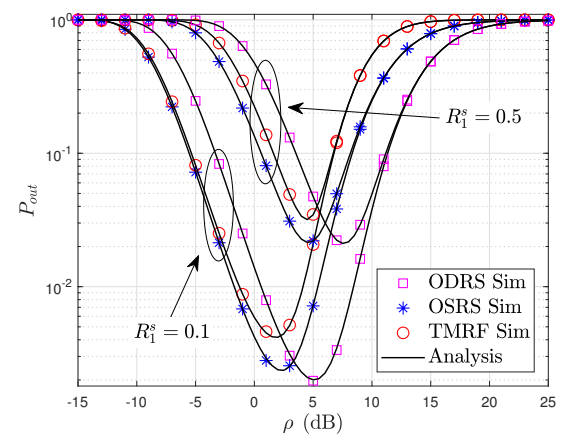

Fig. 4. SOP for various $R_{1}^{s}$ with $R_{2}^{s}=0.2, K=2, m_{U}=m_{E}=m_{R}=2$, $\alpha_{1}=0.2, \alpha_{\mathrm{J}}=0.5, \Omega_{1}=12 \mathrm{~dB}, \Omega_{R}=\Omega_{2}=10 \mathrm{~dB}$, and $\Omega_{E}=-5 \mathrm{~dB}$.

'Sim' denotes the simulation result: 5 , One can observe that the analysis results match perfectly with simulation results and the secrecy performance with OSRS always outperforms that of TMRF and in some scenarios the ODRS scheme can obtain the optimal secrecy outage performance.

Figs. 2 - 6 present the SOP for various $\rho$ with FPA. One can easily observe that the secrecy performance of cooperative NOMA systems is enhanced and then becomes worse by improving the transmit SNR, which is similar to the conclusions in [29] and [30]. This is because the SINR of $U_{2}$ has a ceiling in the higher- $\rho$ region. It is demonstrated in Fig. 2 that the SOP is enhanced by increasing $K$ due to the improved diversity of the cooperative NOMA systems. One can also find that the

${ }^{5}$ We omit the results for TSRS scheme since it is exactly the same as that for OSRS.

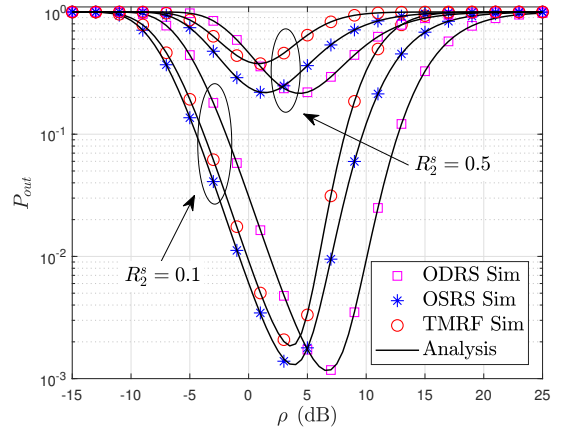

Fig. 5. SOP for various $R_{2}^{s}$ with $R_{1}^{s}=0.2, K=2, m_{U}=m_{E}=m_{R}=2$, $\alpha_{1}=0.2, \alpha_{\mathrm{J}}=0.5, \Omega_{1}=12 \mathrm{~dB}, \Omega_{R}=\Omega_{2}=10 \mathrm{~dB}$, and $\Omega_{E}=-5 \mathrm{~dB}$.

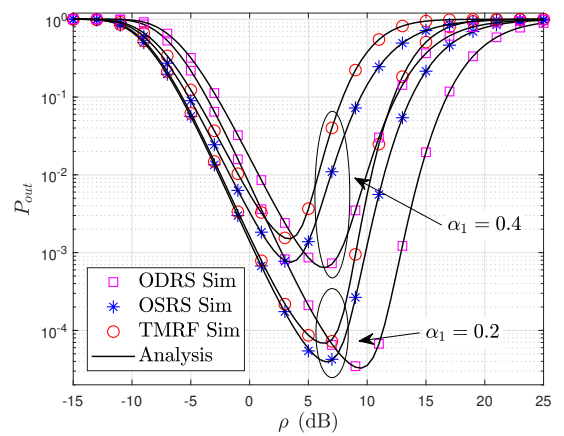

Fig. 6. SOP for various $\alpha_{1}$ with $R_{1}=0.1, R_{2}=0.2, K=2, m_{U}=$ $m_{E}=m_{R}=2, \alpha_{\mathrm{J}}=0.5, \Omega_{1}=12 \mathrm{~dB}, \Omega_{R}=\Omega_{2}=10 \mathrm{~dB}$, and $\Omega_{E}=-10 \mathrm{~dB}$.

SOP depends merely on the RS scheme and is independent of $K$ in the high- $\rho$ region. Fig. 3 demonstrates that the SOP with larger $m$ outperforms the ones with smaller $m$ as larger $m$ signifies that the quality of wireless channel is better. However, the SOP with larger $m$ underperforms that with the smaller $m$ in the high- $\rho$ region. The reason is that the SOP of cooperative NOMA systems will benefit from the stronger fading over eavesdropping channels when the SINR of $U_{2}$ approaches the upper bound.

Figs. 4 and 5 present the effect of different $R_{1}^{s}$ and $R_{2}^{s}$ on SOP of cooperative NOMA systems, respectively. As we know, increasing the secrecy rate threshold deteriorates the secrecy outage performance of wireless communications. Now, from Figs. 4 and 5, we can observe that $R_{1}^{s}$ has significant effect on the SOP of NOMA systems in low- $\rho$ region. Different $R_{1}^{s}$ has significant effect on the SOP of NOMA systems in low- $\rho$ region but does not influence the SOP in high- $\rho$ region. The opposite effect is observed for $R_{2}^{s}$. This proves that in the low- $\rho$ region the bottleneck in secrecy performance of NOMA systems is due to $U_{1}$. In high- $\rho$ region, weaker user must be given more attention. One can also observe from Fig. 4 that the gap between two RS schemes in high- $\rho$ region is larger than those in low- $\rho$ region and the gap in low- $\rho$ region is decreased as $R_{1}^{s}$ decreases.

The impact of PA parameter on the SOP is investigated in Figs. 6- 8. We can observe that the SOP decreases as $\alpha_{1}$ decreases. In other words, the secrecy performance is enhanced by enlarging the difference between allocated power to the two users, which is consistent with the results presented in 


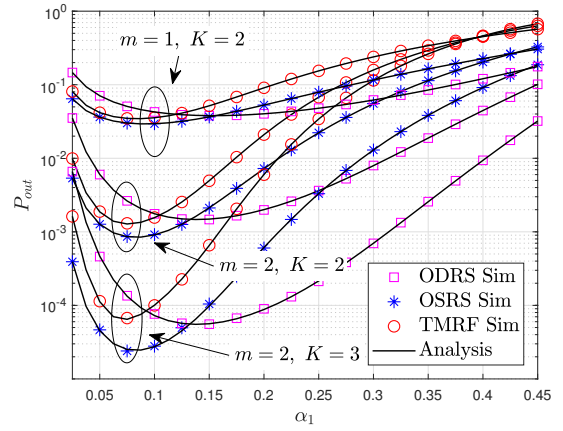

Fig. 7. SOP for various $\alpha_{1}$ and $K$ with $R_{1}=0.1, R_{2}=0.2, m_{U}=$ $m_{E}=m_{R}=m, \alpha_{\mathrm{J}}=0.5, \rho=10 \mathrm{~dB}, \Omega_{1}=12 \mathrm{~dB}, \Omega_{R}=\Omega_{2}=10$ $\mathrm{dB}$, and $\Omega_{E}=-5 \mathrm{~dB}$.

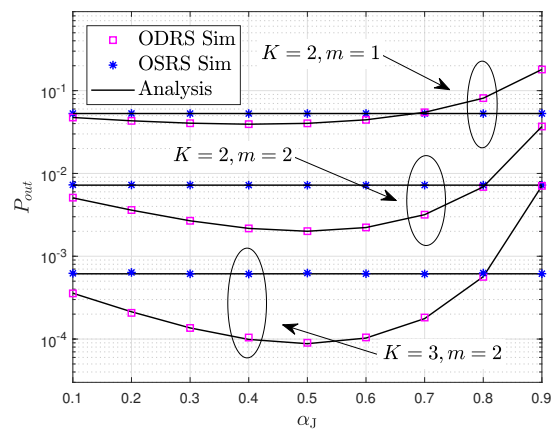

Fig. 8. SOP for various $\alpha_{\mathrm{J}}$ and $K$ and $m$ with $R_{1}=0.1, R_{2}=0.2, \rho=10$ $\mathrm{dB}, m_{U}=m_{E}=m_{R}=m, \alpha_{1}=0.2, \Omega_{1}=12 \mathrm{~dB}, \Omega_{R}=\Omega_{2}=10 \mathrm{~dB}$, and $\Omega_{E}=-5 \mathrm{~dB}$.

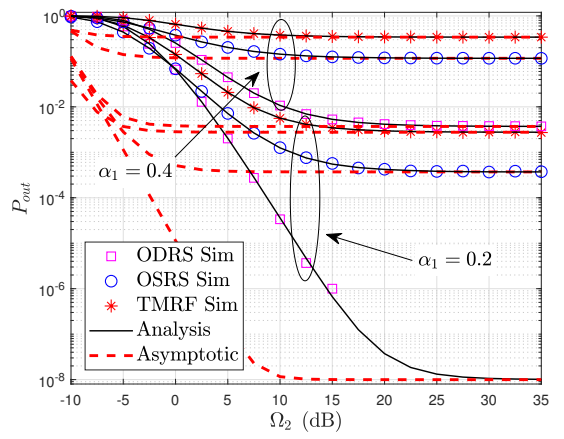

Fig. 9. SOP for various $\alpha_{1}$ with $R_{1}=0.1, R_{2}=0.2, K=2, \alpha_{\mathrm{J}}=0.5$, $\rho=10 \mathrm{~dB}, m_{U}=m_{E}=m_{R}=2, \varepsilon_{1}=1.5, \varepsilon_{2}=2$, and $\Omega_{E}=-10$ dB.

Figs. 4 and 5 . Fig. 8 presents the SOP for various $\alpha_{\mathrm{J}}$. One can observe that the ODRS scheme enhances the secrecy outage performance of cooperative NOMA systems relative to OSRS scheme because the SNR at eavesdropper is degraded. Furthermore, the SOP is firstly improved with increasing $\alpha_{\mathrm{J}}$. However, it gets worse with increasing $\alpha_{\mathrm{J}}$ since the transmit power is reduced. Obviously, there is an optimal $\alpha_{\mathrm{J}}$, which can be easily obtained via one dimensional search based on the results of our work.

The SOP for various $\Omega_{2}$ with FPA is presented in Figs. 9 - 11. One can observe that the asymptotic SOP approaches the exact ones at high- $\Omega_{2}$ region. The SDO of the cooperative NOMA systems is zero since the SOP will be a constant as $\Omega_{2}$ increases. The results in Figs. 9 and 10 prove that increasing

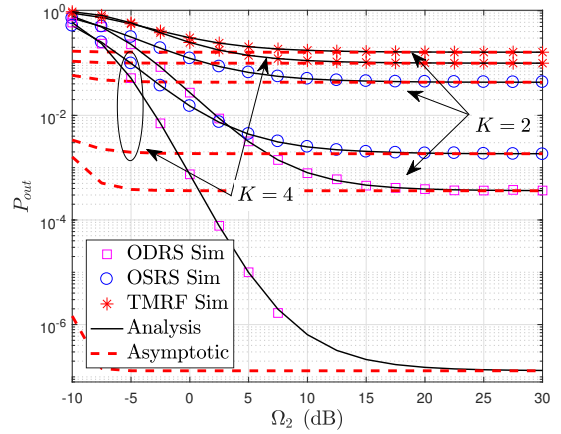

Fig. 10. SOP for various $K$ with $R_{1}=0.1, R_{2}=0.2, m_{U}=m_{E}=$ $m_{R}=2, \rho=15 \mathrm{~dB}, \alpha_{1}=0.2, \alpha_{\mathrm{J}}=0.5, \varepsilon_{1}=1.5, \varepsilon_{2}=2$, and $\Omega_{E}=-12 \mathrm{~dB}$.

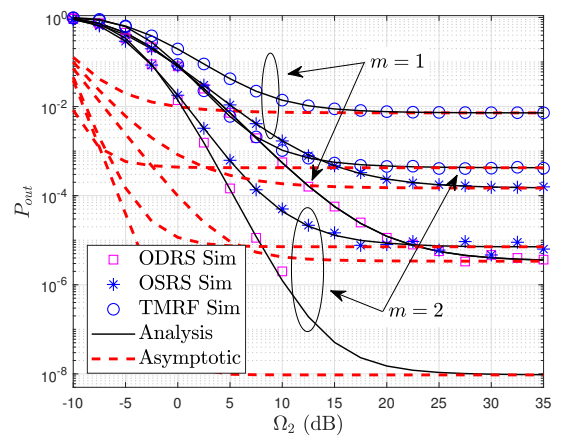

Fig. 11. SOP for various $m$ with $R_{1}=0.1, R_{2}=0.2, m_{U}=m_{E}=$ $m_{R}=m, \alpha_{\mathrm{J}}=0.3, K=3, \rho=10 \mathrm{~dB}, \varepsilon_{1}=1.5, \varepsilon_{2}=2$, and $\Omega_{E}=-10 \mathrm{~dB}$.

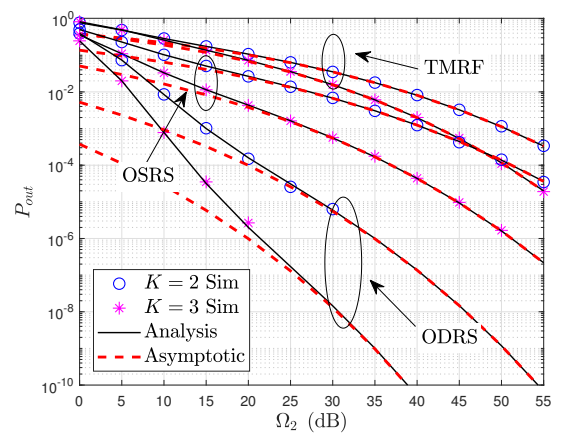

Fig. 12. SOP with DPA scheme for various $K$ with $R_{1}=0.1, R_{2}=0.2$, $\alpha_{\mathrm{J}}=0.5, \rho=10 \mathrm{~dB}, m_{U}=m_{E}=m_{R}=2, \varepsilon_{1}=1.5, \varepsilon_{2}=2$, $\varpi=0.1, \mu=5$, and $\Omega_{E}=-5 \mathrm{~dB}$.

$K$ and / or decreasing $\alpha_{1}$ enhances the SOP of cooperative NOMA systems. The results in Fig. 11 demonstrate that we can obtain better secrecy outage performance under a better channel condition for both TMRF and OSRS schemes.

Figs. 12 - 15 present the SOP for various $\Omega_{2}$ under DPA scheme. One can observe that DPA scheme achieves a nonzero secrecy diversity order, which depends on the relay number, the fading parameters of $S-R_{k}$ and $S_{k}-U_{i}$. Figs. 14 and 15 testify that the SDO of cooperative NOMA systems equal to the minimum SDO of two hops. One can observe from Fig. 15 that a single relay is utilized to send jamming signals influences the SDO of the cooperative NOMA system in some scenarios. 


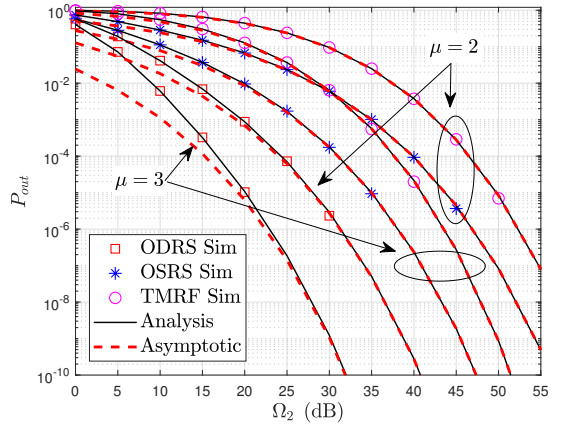

Fig. 13. SOP with DPA scheme for various $\mu$ with $R_{1}=0.1, R_{2}=0.2$, $\alpha_{\mathrm{J}}=0.5, \rho=10 \mathrm{~dB}, m_{U}=m_{E}=m_{R}=2, K=3, \varepsilon_{1}=1.5, \varepsilon_{2}=2$, $\varpi=0.2$, and $\Omega_{E}=-5 \mathrm{~dB}$.

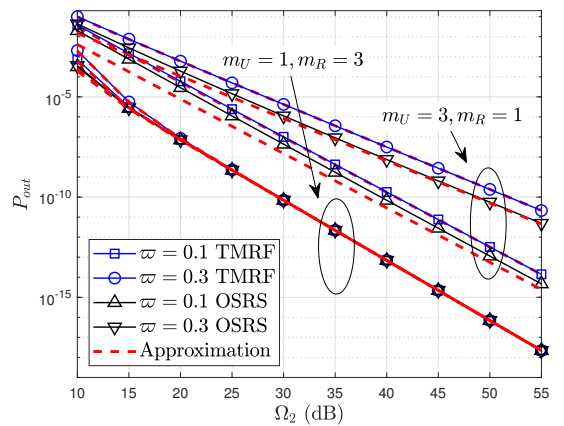

Fig. 14. Asymptotic SOP for TMRF-DPA and OSRS-DPA scheme with $R_{1}=$ $0.1, R_{2}=0.2, \alpha_{\mathrm{J}}=0.5, K=3, \rho=0 \mathrm{~dB}, m_{E}=2, \varepsilon_{1}=1.5, \varepsilon_{2}=2$, $\mu=8$, and $\Omega_{E}=-5 \mathrm{~dB}$.

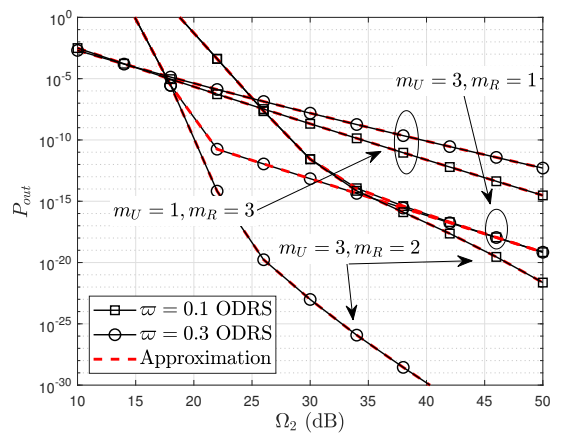

Fig. 15. Asymptotic SOP for ODRS-DPA scheme with $R_{1}=0.1, R_{2}=0.2$, $\alpha_{\mathrm{J}}=0.5, K=3, \rho=10 \mathrm{~dB}, m_{E}=2, \varepsilon_{1}=1.5, \varepsilon_{2}=2, \mu=8$, and $\Omega_{E}=-5 \mathrm{~dB}$.

\section{CONCLUSION}

In this work, three RS schemes were proposed to enhance the secrecy performance of cooperative NOMA systems. We investigated the secrecy outage performance of these schemes under FPA and DPA considering the presence of dependence between the two NOMA users with different secrecy rate thresholds. The analytical expressions for the exact and asymptotic SOP were obtained and verified by Monte Carlo simulation results. Moreover, the SDO and SAG for all the RS schemes were derived. The results demonstrate that relative to TMRF scheme, OSRS and ODRS schemes enhance the security of the cooperative NOMA systems. OSRS scheme achieves the same SDO as TMRF scheme. The jamming signal influences the SDO of ODRS scheme. There are optimal PA coefficients between jamming and signal transmissions, which can be easily obtained via one dimensional search based on the results of our work.

\section{APPENDIX A}

The PDF of $H_{E}=\max _{k \in \bar{\Phi}}\left\{G_{E}^{k}\right\}$ is obtained as

$$
f_{H_{E}}(z)=(K-n)\left(F_{G_{E}}(z)\right)^{K-n-1} f_{G_{E}}(z),
$$

where $F_{G_{E}}(z)$ and $f_{G_{E}}(z)$ refer to the CDF and PDF, respectively, of $G_{E}^{k}$, which is easily obtained by utilizing (11). Based on multinomial theorem, we obtain the PDF of $H_{E}$ as

$$
f_{H_{E}}(z)=\frac{(K-n) \lambda_{E}^{m_{E}}}{\Gamma\left(m_{E}\right)} \sum_{S_{E}} A z^{B+m_{E}-1} e^{-C \lambda_{E} z},
$$

$$
\begin{aligned}
& \text { where } S_{E}=\left\{\left(n_{1}, \cdots, n_{m_{E}+1}\right) \in \mathbb{N}\left|\sum_{p=1}^{m_{E}+1} n_{p}=K-n-1\right|\right\}, \\
& A=\left(\frac{(K-n-1) !}{\prod_{q=1}^{m_{E}+1}\left(n_{q}\right) !}\right) \prod_{p=2}^{m_{E}+1}\left(-\frac{\lambda_{E}^{p-2}}{(p-2) !}\right)^{n_{p}}, \quad B=
\end{aligned}
$$$$
\sum_{p=2}^{m_{E}+1} n_{p}(p-2) \text {, and } C=1+\sum_{p=2}^{m_{E}+1} n_{p} \text {. }
$$

\section{APPENDIX B}

Based on (32) and utilizing (1.111) and (3.351.3) of [50], the CDF of $Y$ can be obtained as (59) at the bottom of the next page, where $\varphi=\frac{(K-n) \lambda_{E}^{m_{E}}}{\Gamma\left(m_{E}\right)}, \varsigma=B+m_{E}+j$, and $\delta=\frac{C_{k}^{j} A \rho_{4}^{j} \lambda_{E}^{k-\varsigma}(\varsigma-1) !}{k !}$.

Using integration by parts, and applying some algebra, we derive the PDF of $Y$ as 33 .

\section{APPENDIX C}

Based on $\alpha_{1}^{\mathrm{DPA}}$ and $\alpha_{2}^{\mathrm{DPA}}$, we have $a_{i}^{\mathrm{DPA}}=$ $\frac{\left(1+\mu \lambda_{2}^{-\varpi}\right)^{2}-\theta_{2} \mu \lambda_{2}^{-\varpi}-\theta_{2}}{\theta_{2} \mu \lambda_{2}^{-\varpi} \rho_{i}}, b_{i}^{\mathrm{DPA}}=\frac{\theta_{1}-1}{\rho_{i}}\left(1+\mu \lambda_{2}^{-\varpi}\right), c_{i}^{\mathrm{DPA}}=$ $-\frac{1+\mu \lambda_{2}^{-\varpi}}{\rho_{i}}, d_{i}^{\mathrm{DPA}}=\frac{\left(1+\mu \lambda_{2}^{-\varpi}-\theta_{2}\right) \rho_{i}}{\left(1+\mu \lambda_{2}^{-\varpi}\right)^{2}}, e_{i}^{\mathrm{DPA}}=\frac{\theta_{2} \mu \lambda_{2}^{-\varpi} \rho_{i}^{2}}{\left(1+\mu \lambda_{2}^{-\varpi}\right)^{3}}$, and $i=1,2$. Due to $\lambda_{2}^{-\varpi}=\frac{\Omega_{2}^{\varpi}}{m_{U}^{\varpi}} \sim \Omega_{2}^{\varpi}$, one can easily have $\alpha_{1}^{\mathrm{DPA}}=\frac{1}{1+\mu \lambda_{2}^{-\varpi}} \sim \frac{1}{\mu} \Omega_{2}^{-\varpi}, \alpha_{2}^{\mathrm{DPA}}=\frac{\mu \lambda_{2}^{-\varpi}}{1+\mu \lambda_{2}^{-\varpi}} \sim \Omega_{2}^{0}$, $a_{i}^{\mathrm{DPA}} \sim \frac{\mu}{\theta_{2} \rho_{i} m_{U}^{\varpi}} \Omega_{2}^{\varpi}, b_{i}^{\mathrm{DPA}} \sim \frac{\theta_{1}-1}{\rho_{i}} \mu \Omega_{2}^{\varpi}, c_{i}^{\mathrm{DPA}} \sim-\frac{\mu}{\rho_{i}} \Omega_{2}^{\varpi}$, $d_{i}^{\text {DPA }} \sim \frac{\rho_{i}}{\mu} \Omega_{2}^{-\varpi}$, and $e_{i}^{\text {DPA }} \sim \frac{\theta_{2} m_{U}^{2 \varpi} \rho_{i}^{2}}{\mu^{2}} \Omega_{2}^{-2 \varpi}$. Then substituting these variables into (45), it can be found that the SDO of $I_{j}^{\mathrm{TMRF}}(j=1,3,4)$ is infinity. In other words, The SDO of the second hop under TMRF scheme depends on $I_{2}^{\mathrm{TMRF}}$, which is given as

$$
\begin{aligned}
I_{2}^{\mathrm{TMRF}} & =\frac{\beta_{E}^{\mathrm{TMRF}} m_{U}^{\tau_{U}}}{\left(\varepsilon_{1} \Omega_{2}\right)^{\tau_{U}}\left(\tau_{U}\right) !} \sum_{k=0}^{\tau_{U}} \frac{C_{\tau_{U}}^{k} \theta_{1}^{k}}{\lambda_{E}^{k+\tau_{E}}} \Upsilon\left(k+\tau_{E}, \lambda_{E} a_{1}^{\mathrm{DPA}}\right) \\
& \times\left(\frac{\left(\theta_{1}-1\right)\left(1+\mu \lambda_{2}^{-\varpi}\right)}{\rho_{i}}\right)^{\tau_{U}-k} \\
& \approx \sum_{k=0}^{\tau_{U}} \frac{C_{\tau_{U}}^{k} \theta_{1}^{k} m_{U}^{\tau_{U}}\left(m_{U}\right)^{-\varpi\left(\tau_{U}-k\right)}}{\Gamma\left(\tau_{E}\right) \lambda_{E}^{k} \varepsilon_{1}^{\tau_{U}}\left(\tau_{U}\right) !}\left(\frac{\mu\left(\theta_{1}-1\right)}{\rho_{i}}\right)^{\tau_{U}-k} \\
& \times \Upsilon\left(k+\tau_{E}, \lambda_{E} a_{1}^{\mathrm{DPA}}\right) \Omega_{2}^{-\left(\tau_{U}-\varpi\left(\tau_{U}-k\right)\right)} .
\end{aligned}
$$


Thus, we obtain the SDO and SAG of $I_{2}^{\mathrm{TMRF}}$ as

$$
\begin{aligned}
G_{d, 2}^{\mathrm{TMRF}} & =\min _{0 \leq k \leq \tau_{U}}\left\{\tau_{U}-\varpi\left(\tau_{U}-k\right)\right\} \\
& =\tau_{U}(1-\varpi), \\
G_{a, 2}^{\mathrm{TMRF}} & =\frac{m_{U}^{\tau_{U}(1-\varpi)}}{\varepsilon_{1}^{\tau_{U}}\left(\tau_{U}\right) !}\left(\frac{\mu\left(\theta_{1}-1\right)}{\rho_{i}}\right)^{\tau_{U}},
\end{aligned}
$$

respectively.

Substituting $\varphi_{R}$ into 38 , we obtain

$$
\begin{aligned}
P_{\mathrm{out}}^{\infty}= & \sum_{n=0}^{K} C_{K}^{n} \varphi_{R}^{K-n} \eta^{m_{R}(K-n)} P_{\Phi_{n}}^{\infty} \\
= & \sum_{n=0}^{K} C_{K}^{n}\left(\frac{m_{R}^{m_{R}}}{\varepsilon_{2}^{m_{R}}\left(m_{R}\right) !}\right)^{K-n} \\
& \quad \times \eta^{m_{R}(K-n)} \Omega_{2}^{-m_{R}(K-n)} P_{\Phi_{n}}^{\infty} .
\end{aligned}
$$

Then the SDO of the cooperative NOMA systems in this case is obtained as

$$
\begin{aligned}
G_{d}^{\mathrm{TMRF}, \mathrm{DPA}} & =\min _{0 \leq n \leq K}\left\{m_{R}(K-n)+\tau_{U}(1-\varpi)\right\} \\
& =K \min \left\{m_{U}(1-\varpi), m_{R}\right\} .
\end{aligned}
$$

The SAG of cooperative NOMA systems under TMRF scheme is obtained as (47).

\section{REFERENCES}

[1] L. Dai, B. Wang, Y. Yuan, S. Han, C.-L. I, and Z. Wang, "Non-orthogonal multiple access for 5G: solutions, challenges, opportunities, and future research trends," IEEE Commun. Mag., vol. 53, no. 9, pp. 74-81, Sept. 2015.

[2] Y. Wang, B. Ren, S. Sun, S. Kang and X. Yue, "Analysis of nonorthogonal multiple access for 5G," China Communications, vol. 13, no. Supplement2, pp. 52-66, N/A 2016.

[3] S. M. R. Islam, N. Avazov, O. A. Dobre, and K. S. Kwak, "Power-domain non-orthogonal multiple access (NOMA) in 5G systems: Potentials and challenges," IEEE Commun. Surveys Tuts., vol. 19, no. 2, pp. 721 - 742, 2nd Quart. 2017.

[4] Y. Liu, H. Xing, C. Pan, A. Nallanathan, M. Elkashlan, and L. Hanzo, "Multiple antenna assisted non-orthogonal multiple access," IEEE Wireless Commun., vol. 25, no. 2, pp. 17 - 23, Apr. 2018

[5] D. Wan, M. Wen, F. Ji, H. Yu, and F. Chen, "Non-orthogonal multiple access for cooperative communications: Challenges, opportunities, and trends," IEEE Wireless Commun., vol. 25, no. 2, pp. 109-117, Apr. 2018.

[6] F. Zhou, Y. Wu, Y.-C. Liang, Z. Li, Y. Wang, and K.-K. Wong, "State of the art, taxonomy, and open issues on cognitive radio networks with NOMA," IEEE Wireless Commun., vol. 25, no. 2, pp. 100-108, Apr. 2018.
[7] Z. Ding, M. Peng, and H. V. Poor, "Cooperative non-orthogonal multiple access in 5G systems," IEEE Commun. Lett., vol. 19, no. 8, pp. 14621465, Aug. 2015.

[8] Y. Liu, Z. Ding, M. Elkashlan, and H. V. Poor, "Cooperative nonorthogonal multiple access with simultaneous wireless information and power transfer," IEEE J. Sel. Areas Commun., vol. 34, no. 4, pp. 938953, Apr. 2016.

[9] J. Men and J. Ge, "Non-orthogonal multiple access for multiple-antenna relaying networks," IEEE Commun. Lett., vol. 19, no. 10, pp. 1686-1689, Oct. 2015.

[10] J. Men, J. Ge, and C. Zhang, "Performance analysis of non-orthogonal multiple access for relaying networks over Nakagami- $m$ fading channels," IEEE Trans. Veh. Technol., vol. 66, no. 2, pp. 1200-1208, Feb. 2017.

[11] Y. Xiao, L. Hao, Z. Ma, Z. Ding, Z. Zhang, and P. Fan, "Forwarding strategy selection in dual-hop NOMA relaying systems," IEEE Commun. Lett., vol. 22, no. 8, pp. 1644-1647, Aug. 2018.

[12] W. Duan, M. Wen, Z. Xiong, and M. H. Lee, "Two-stage power allocation for dual-hop relaying systems with non-orthogonal multiple access," IEEE Access, vol. 5, pp. 2254-2261, Mar. 2017.

[13] L. Lv, J. Chen, Q. Ni, and Z. Ding, "Design of cooperative nonorthogonal multicast cognitive multiple access for 5G systems: User scheduling and performance analysis," IEEE Trans. Commun., vol. 65, no. 6, pp. 2641-2656, Mar. 2017.

[14] L. Lv, J. Chen, Q. Ni, Z. Ding, and H. Jiang, "Cognitive non-orthogonal multiple access with cooperative relaying: A new wireless frontier for $5 \mathrm{G}$ spectrum sharing," IEEE Commun. Mag., vol. 56, no. 4, pp. 188-195, Apr. 2018.

[15] C. Zhong and Z. Zhang, "Non-Orthogonal multiple access with cooperative full-duplex relaying," IEEE Commun. Lett., vol. 20, no. 12, pp. 2478-2481, Dec. 2016.

[16] Z. Zhang, Z. Ma, M. Xiao, Z. Ding, and P. Fan, "Full-duplex device-todevice aided cooperative non-orthogonal multiple access," IEEE Trans. Veh. Technol., vol. 66, no. 5, pp. 4467 - 4471, May 2016.

[17] G. Liu, X. Chen, Z. Ding, Z. Ma, and F. R. Yu, "Hybrid half-duplex/fullduplex cooperative non-orthogonal multiple access with transmit power adaptation," IEEE Trans. Wireless Commun., vol. 17, no. 1, pp. 506-519, Jan. 2018.

[18] T. M. C. Chu and H.-J. Zepernick, "Performance of a non-orthogonal multiple access system with full-duplex relaying," IEEE Commun. Lett., vol. 22, no. 10, pp. 2084-2087, Oct. 2018.

[19] M. F. Kader, S. Y. Shin, and V. C. M. Leung, "Full-duplex nonorthogonal multiple access in cooperative relay sharing for 5G systems," IEEE Trans. Veh. Technol., vol. 67, no. 6, pp. 5831-5840, Jul. 2018.

[20] Z. Ding, H. Dai, and H. V. Poor, "Relay selection for cooperative NOMA,” IEEE Wireless Commun. Lett., vol. 5, no. 4, pp. 416-419, Aug. 2016.

[21] J. Zhao, Z. Ding, P. Fan, Z. Yang, and G. K. Karagiannidis, "Dual relay selection for cooperative NOMA with distributed space time coding," IEEE Access, vol. 6, pp. 20440-20450, Apr. 2018.

[22] Z. Yang, Z. Ding, Y. Wu, and P. Fan, "Novel relay selection strategies for cooperative NOMA," IEEE Trans. Veh. Technol., vol. 66, no. 11, pp. 10114-10123, Nov. 2017.

[23] P. Xu, Z. Yang, Z. Ding, and Z. Zhang, "Optimal relay selection schemes for cooperative NOMA," IEEE Trans. Veh. Technol., vol. 67, no. 8, pp. 7851-7855, Aug. 2018.

[24] X. Yue, Y. Liu, S. Kang, A. Nallanathan, and Z. Ding, "Spatially random

$$
\begin{aligned}
F_{Y}(y) & =\operatorname{Pr}\left\{\frac{G_{E}^{k}}{1+\rho_{4} H_{E}}<y\right\} \\
& =\operatorname{Pr}\left\{G_{E}^{k}<y+\rho_{4} H_{E} y\right\} \\
& =\int_{0}^{\infty} F_{G_{E}^{k}}\left(y+\rho_{4} x y\right) f_{H_{E}}(x) d x \\
& =1-\varphi \sum_{k=0}^{m_{E}-1} \sum_{S_{E}} \sum_{j=0}^{k} \frac{C_{k}^{j} A \rho_{4}^{j} \lambda_{E}^{k} e^{-\lambda_{E} y} y^{k}}{k !} \int_{0}^{\infty} x^{B+j+m_{E}-1} e^{-\lambda_{E}\left(C+\rho_{4} y\right) x} d x \\
& =1-\varphi \sum_{k=0}^{m_{E}-1} \sum_{S_{E}} \sum_{j=0}^{k} \frac{\delta e^{-\lambda_{E} y} y^{k}}{\left(C+\rho_{4} y\right)^{\varsigma}}
\end{aligned}
$$


relay selection for full/half-duplex cooperative NOMA networks," IEEE Trans. Commun., vol. 66, no. 8, pp. 3294 - 3308, Aug. 2018.

[25] J. Chen, L. Yang, and M.-S. Alouini, "Performance analysis of cooperative NOMA schemes in spatially random relaying networks," IEEE Access, vol. 6, pp. 33159-33168, Jul. 2018.

[26] M. Bloch, J. Barros, M. R. D. Rodrigues, and S. W. McLaughlin, "Wireless information-theoretic security," IEEE Trans. Inf. Theory, vol. 54, no. 6, pp. 2515-2534, Jun. 2008.

[27] Y. Zhang, H. Wang, Q. Yang, and Z. Ding, "Secrecy sum rate maximization in non-orthogonal multiple access," IEEE Commun. Lett., vol. 20, no. 5, pp. 930-933, May. 2016.

[28] Y. Liu, Z. Qin, M. Elkashlan, Y. Gao, and L. Hanzo, "Enhancing the physical layer security of non-orthogonal multiple access in large-scale networks," IEEE Trans. Wireless Commun., vol. 16, no. 3, pp. 1656-1672, Mar. 2017.

[29] H. Lei, J. Zhang, K.-H. Park, P. Xu, I. S. Ansari, G. Pan, B. Alomair, and M.-S. Alouini, "On secure NOMA systems with transmit antenna selection schemes," IEEE Access, vol. 5, pp. 17450-17464, Sep. 2017.

[30] H. Lei, J. Zhang, K.-H. Park, P. Xu, Z. Zhang, G. Pan, and M.-S. Alouini, "Secrecy outage of MAX-MIN TAS scheme in MIMO-NOMA systems," IEEE Trans. Veh. Technol., vol. 67, no. 8, pp. 6981-6990, Aug. 2018.

[31] Y. Feng, S. Yan, and Z. Yang, "Secure transmission to the strong user in non-orthogonal multiple access," IEEE Commun. Lett., vol. 22, no. 12, pp. 2623-2626, Dec. 2018.

[32] L. Lv, Z. Ding, Q. Ni, and J. Chen, "Secure MISO-NOMA transmission with artificial noise," IEEE Trans. Veh. Technol., vol. 67, no. 7, pp. 67006705, Jul. 2018.

[33] F. Zhou, Z. Chu, H. Sun, R. Q. Hu, and L. Hanzo, "Artificial noise aided secure cognitive beamforming for cooperative MISO-NOMA using SWIPT," IEEE J. Sel. Areas Commun., vol. 36, no. 4, pp. 918-931, Apr. 2018.

[34] N. Zhao, W. Wang, J. Wang, Y. Chen, Y. Lin, Z. Ding, and N. C. Beaulieu, "Joint beamforming and jamming optimization for secure transmission in MISO-NOMA networks," IEEE Trans. Commun., vol. 67, no. 3, pp. 2294-2305, Mar. 2019.

[35] Z. Zhu, Z. Chu, F. Zhou, H. Niu, Z. Wang, and I. Lee, "Secure beamforming designs for secrecy MIMO SWIPT systems," IEEE Wireless Commun. Lett., vol. 7, no. 3, pp. 424-427, Jun. 2018.

[36] K. Jiang, T. Jing, Y. Huo, F. Zhang, and Z. Li, "SIC-based secrecy performance in uplink NOMA multi-eavesdropper wiretap channels," IEEE Access, vol. 6, pp. 19664-19680, Apr. 2018.

[37] H. Zhang, N. Yang, K. Long, M. Pan, G. K. Karagiannidis, and V. C. M. Leung, "Secure communications in NOMA system: Subcarrier assignment and power allocation," IEEE J. Sel. Areas Commun., vol. 36, no. 7, pp. 1441-1452, Jul. 2018.

[38] J. Chen, L. Yang, and M.-S Alouini, "Physical layer security for cooperative NOMA systems," IEEE Trans. Veh. Technol., vol. 67, no. 5, pp. 4645-4649, May. 2018.

[39] W. Jiang, Y. Gong, Q. Xiao, and Y. Liao, "Rate maximization for untrusted relay networks with nonorthogonal cooperative transmission protocols," IEEE Trans. Veh. Technol., vol. 67, no. 7, pp. 6325-6339, Jul. 2018

[40] A. Arafa, W. Shin, M. Vaezi, and H. V. Poor, "Secure relaying in non-orthogonal multiple access: Trusted and untrusted Scenarios," IEEE Transactions on Information Forensics and Security, ( Early Access ), doi: 10.1109/TIFS.2019.2911162, Apr. 2019.

[41] Y. Feng, Z. Yang, and S. Yan, "Non-orthogonal multiple access and artificial-noise aided secure transmission in FD relay networks," in Proc. 2017 IEEE Globecom Workshops (GC Wkshps), Singapore, Dec. 2017, pp. 1-6.

[42] C. Liu, L. Zhang, M. Xiao, Z. Chen, and S. Li, "Secrecy performance analysis in downlink NOMA systems with cooperative full-duplex relaying," in Proc. 2018 IEEE International Conference on Communications Workshops (ICC Workshops), Kansas City, MO, USA, Jul. 2018, pp. 1-6.

[43] B. Li, X. Qi, K. Huang, Z. Fei, F. Zhou, and R. Q. Hu, "Securityreliability tradeoff analysis for cooperative NOMA in cognitive radio networks," IEEE Trans. Commun., vol. 67, no. 1, pp. 83-96, Jan. 2019.

[44] Y. Zou, X. Wang, and W. Shen, "Optimal relay selection for physicallayer security in cooperative wireless networks," IEEE J. Sel. Areas Commun., vol. 31, no. 10, pp. 2099-2111, Oct. 2013.

[45] L. Fan, X. Lei, N. Yang, T. Q. Duong, and G. K. Karagiannidis, "Secure multiple amplify-and-forward relaying with cochannel interference," IEEE J. Sel. Topics Signal Process., vol. 10, no. 8, pp. 1494-1505, Dec. 2016.

[46] L. Fan, R. Zhao, F.-K. Gong, N. Yang, and G. K. Karagiannidis, "Secure multiple amplify-and-forward relaying over correlated fading channels," IEEE Trans. Commun., vol. 65, no. 7, pp. 2811-2820, Jul. 2017.
[47] R. Zhao, Y. Yuan, L. Fan, and Y. C. He, "Secrecy performance analysis of cognitive decode-and-forward relay networks in Nakagami- $m$ fading channels," IEEE Trans. Commun., vol. 65, no. 2, pp. 549-563, Feb. 2017.

[48] H. Lei, H. Zhang, I. S. Ansari, Z. Ren, G. Pan, K. A. Qaraqe, and M.S. Alouini, "On secrecy outage of relay selection in underlay cognitive radio networks over Nakagami- $m$ fading channel," IEEE Trans. Cogn. Commun. Netw., vol. 3, no. 4, pp. 614 - 627, Dec. 2017.

[49] H. Lei, Z. Yang, K.-H. Park, I. S. Ansari, G. Pan, and M.-S. Alouini, "On physical layer security of multiple-relay assisted NOMA systems," 2019 IEEE International Conference on Communications Workshops (ICC Workshops): Non-Orthogonal Multiple Access Techniques for 5G, Shanghai, May 2019, pp 1-6.

[50] I. S. Gradshteyn and I. M. Ryzhik, Table of Integrals, Series, and Products: Academic press, 2014.

[51] H. Lei, C. Gao, I. S. Ansari, Y. Guo, Y. Zou, G. Pan, and K. Qaraqe, "Secrecy outage performance of transmit antenna selection for MIMO underlay cognitive radio systems over Nakagami- $m$ channels," IEEE Trans. Veh. Technol., vol. 66, no. 3, pp. 2237-2250, Mar. 2017.

[52] M. Abramowitz and I. A. Stegun, Handbook of Mathematical Functions with Formulas, Graphs, and Mathematical Tables, 9th. New York, NY, USA: Dover Press, 1972.

[53] L. Dong, Z. Han, A. P. Petropulu, and H. V. Poor, "Improving wireless physical layer security via cooperating relays," IEEE Trans. Signal Process., vol. 58, no. 3, pp. 1875-1888, Mar. 2010.

[54] Y. Zou, J. Zhu, X. Wang, and V. Leung, "Improving physical-layer security in wireless communications using diversity techniques," IEEE Netw., vol. 29, no. 1, pp. 42-48, Jan. 2015.

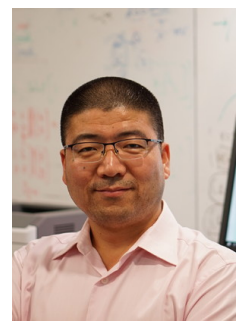

Hongjiang Lei (M'17) received the B.Sc. degree in Mechanical and Electrical Engineering from Shenyang Institute of Aeronautical Engineering, Shenyang, China, in 1998, the M.Sc. degree in Computer Application Technology from Southwest Jiaotong University, Chengdu, China, in 2004, and the $\mathrm{Ph} . \mathrm{D}$. degree in Instrument Science and Technology from Chongqing University, Chongqing, China, in 2015, respectively. In May 2004, he joined the School of Communication and Information Engineering (SCIE) of Chongqing University of Posts and Telecommunications (CQUPT), Chongqing, China, where he is currently an Associate Professor. From November 2016 to October 2018, he was a Postdoctoral Research Fellow under the supervision of Prof. MohamedSlim Alouini with CEMSE Division, King Abdullah University of Science and Technology (KAUST), Saudi Arabia. His current research interests include physical layer security, cooperative relaying systems, and cognitive radio networks. He is a TPC Member of IEEE Globecom'2017, 2018, 2019, etc. $\mathrm{He}$ has also served as a reviewer for major international journals, e.g., IEEE JSAC, IEEE JSTSP, IEEE TVT, IEEE TCOM, IEEE TWC, IEEE TIFS, IEEE WCL, IEEE CL, etc.

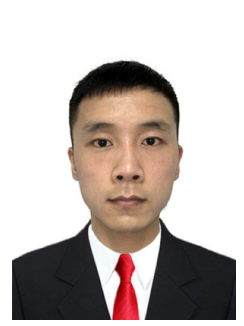

Zixuan Yang received the B.Sc. degree in Electronics and Information Engineering from North China University of Water Resources and Electric Power (NCWU), Zhengzhou, China, in 2015. He is currently pursuing the M.Sc. degree in Information and Communication Engineering at CQUPT, Chongqing, China. His research interests include physical layer security, non-orthogonal multiple access (NOMA) and cooperative communication. 


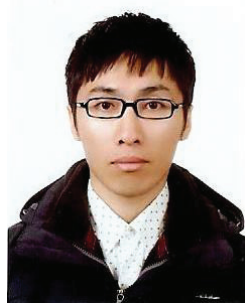

Ki-Hong Park (S'06-M'11) received the B.Sc. degree in electrical, electronic, and radio engineering from Korea University, Seoul, Korea, in 2005 and the M.S. and Ph.D. degrees from the School of Electrical Engineering, Korea University, Seoul, Korea, in 2011. Since April 2011, he has been a Postdoctoral Fellow of Electrical Engineering in the Division of Physical Science and Engineering, King Abdullah University of Science and Technology (KAUST), Thuwal, Saudi Arabia. His research interests are broad in communication theory and its application to the design and performance evaluation of wireless communication systems and networks. On-going research includes the application to MIMO diversity/beamforming systems, cooperative relaying systems, physical layer secrecy, and optical wireless communications.

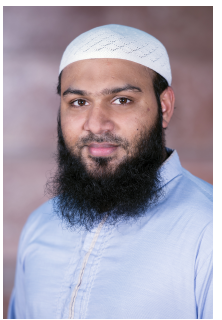

Imran Shafique Ansari (S'07-M'15) received the B.Sc. degree in Computer Engineering from King Fahd University of Petroleum and Minerals (KFUPM) in 2009 (with First Honors) and M.Sc. and $\mathrm{PhD}$ degrees from King Abdullah University of Science and Technology (KAUST) in 2010 and 2015, respectively. Currently, since August 2018, he is a Lecturer (Assistant Professor) with University of Glasgow, Glasgow, UK. Prior to this, from November 2017 to July 2018, he was a Lecturer (Assistant Professor) with Global College of Engineering and Technology (GCET) (affiliated with University of the West of England (UWE), Bristol, UK). From April 2015 to November 2017, he was a Postdoctoral Research Associate (PRA) with Texas A\&M University at Qatar (TAMUQ). From May 2009 through Aug. 2009, he was a visiting scholar with Michigan State University (MSU), East Lansing, MI, USA, and from Jun. 2010 through Aug. 2010, he was a research intern with Carleton University, Ottawa, ON, Canada.

Dr. Ansari has authored/co-authored 70+ journal and conference publications. He has co-organized the GRASNET'2016, 2017, 2018 workshops in conjunction with IEEE WCNC'2016, 2017 and IEEE Globecom 2018. His current research interests include free-space optics (FSO), channel modeling/signal propagation issues, relay/multihop communications, physical layer secrecy issues, full duplex systems, and secure D2D applications for 5G+ systems, among others.

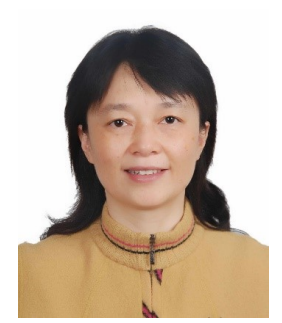

Yongcai Guo received B.Eng degree from Northwest Institute of Telecommunication Engineering in 1982, and received M.Sc. degree in 1988 and Ph.D. in 1999 both from Chongqing University. Now she is a professor in Chongqing University. Her research interests include opto-electronic technology and system, digital signal processing, etc.

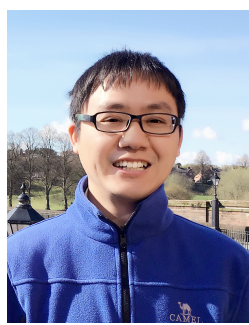

Gaofeng Pan (M'12) received his B.Sc in Communication Engineering from Zhengzhou University, Zhengzhou, China, in 2005, and the Ph.D. degree in Communication and Information Systems from Southwest Jiaotong University, Chengdu, China, in 2011. He was with The Ohio State University, Columbus, OH, USA, from September 2009 to April 2011 as a joint-trained PhD student under the supervision of Prof. Eylem Ekici. From May 2012 to April 2019, he was with Southwest University, Chongqing, China, and he was also with School of Computing and Communications, Lancaster University, Lancaster, U.K., from Jan. 2016 to Jan. 2018, where he was a postdoc under the supervision of Prof. Zhiguo Ding. Since April 2019, he has been with School of Information and Electronics, Beijing Institute of Technology, P. R. China, as a professor. His research interest spans special topics in communications theory, signal processing and protocol design, including visible light communications, secure communications, $\mathrm{CR} /$ cooperative communications, UAV communications and MAC protocols. He has served as a TPC Member of IEEE Globecom, IEEE ICC, and IEEE VTC etc. He has also served as a reviewer for major international journals, e.g., IEEE JSAC, IEEE TCOM, IEEE TWC, IEEE TSP, IEEE TVT, IEEE CL, IEEE WCL, etc.

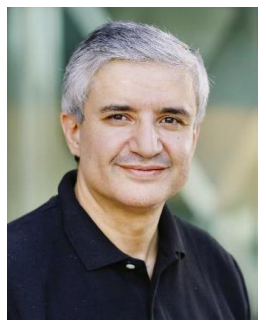

Mohamed-Slim Alouini (S'94-M'98-SM'03-F'09) was born in Tunis, Tunisia. He received the Ph.D. degree in Electrical Engineering from the California Institute of Technology (Caltech), Pasadena, CA, USA, in 1998. He served as a faculty member in the University of Minnesota, Minneapolis, MN, USA, then in the Texas A\&M University at Qatar, Education City, Doha, Qatar before joining King Abdullah University of Science and Technology (KAUST), Thuwal, Makkah Province, Saudi Arabia as a Professor of Electrical Engineering in 2009. His current research interests include the modeling, design, and performance analysis of wireless communication systems. 